\title{
On a free boundary problem describing the phase transition in an incompressible viscous fluid
}

\author{
YOSHIAKI KUSAKA \\ Department of Mathematics, Faculty of Engineering, Tamagawa University, \\ 6-1-1 Tamagawa-Gakuen, Machida, Tokyo 194-8611, Japan \\ E-mail: kusaka@eng.tamagawa.ac.jp
}

[Received 5 January 2009 and in revised form 6 January 2010]

\begin{abstract}
Ice melts at $0^{\circ} \mathrm{C}$ under a pressure of $1 \mathrm{~atm}$, and increasing the pressure decreases the melting temperature. In the present paper, a new problem is posed that describes the process of phase transition in an incompressible viscous fluid, taking into account the above-described pressure effect. This problem is described as a free boundary problem in terms of the Navier-Stokes equations coupled with the heat equation, where the equilibrium temperature is assumed to be related to the pressure by the Clapeyron-Clausius equation. We prove the existence of a global-in-time solution.
\end{abstract}

\section{Introduction}

Let $\Omega_{t}$ be a time-dependent bounded domain in $\mathbb{R}^{3}$. Let the boundary of $\Omega_{t}$ consist of two pieces, namely, a time-dependent piece $\Gamma_{t}$ and a rigid piece $\Sigma$. Let $\Omega_{t}$ represent a liquid region, and $\Gamma_{t}$ represent an interface with another phase. In $\Omega_{t}$, the velocity $v$, the pressure $p$, and the temperature $T$ are assumed to satisfy

$$
\left\{\begin{array}{l}
\nabla \cdot \boldsymbol{v}=0, \quad \frac{\partial \boldsymbol{v}}{\partial t}+(\boldsymbol{v} \cdot \nabla) \boldsymbol{v}+\frac{1}{\rho} \nabla p-v \Delta \boldsymbol{v}=\mathbf{0}, \\
\frac{\partial T}{\partial t}+(\boldsymbol{v} \cdot \nabla) T-\frac{\kappa}{\rho C_{p}} \Delta T=\frac{2 v}{C_{p}} \mathbf{D}(\boldsymbol{v}): \mathbf{D}(\boldsymbol{v}),
\end{array}\right.
$$

where $v, \rho, C_{p}$, and $\kappa$ are the kinematic viscosity, the density, the specific heat at constant pressure, and the heat conductivity, which are assumed to be positive constants, and $\mathbf{D}(\boldsymbol{v})$ is the velocity deformation tensor with elements $(\mathbf{D}(\boldsymbol{v}))_{i j}=\frac{1}{2}\left(\frac{\partial v_{i}}{\partial x_{j}}+\frac{\partial v_{j}}{\partial x_{i}}\right)(i, j=1,2,3)$. Equation (1) includes the Navier-Stokes equations and the heat equation with transport and viscous dissipation terms. On $\Gamma_{t}$, we assume the following conditions, which are derived by applying the laws of conservation of mass, conservation of momentum, and conservation of energy across the interface (see, e.g., [7], [14]):

$$
\left\{\begin{array}{l}
\boldsymbol{v} \cdot \mathbf{n}=\left(1-\frac{\rho_{e}}{\rho}\right) V, \quad \mathbf{T}(\boldsymbol{v}, p) \mathbf{n}=\left[\boldsymbol{v}(\boldsymbol{v}-V \mathbf{n})^{t}\right] \mathbf{n}-p_{m} \mathbf{n}, \\
l \rho_{e} V=-\kappa \nabla T \cdot \mathbf{n}, \quad T=T_{m},
\end{array}\right.
$$

where $\rho_{e}$ and $l$ are the density of the solid and the latent heat, which are assumed to be positive constants, $T_{m}$ is the equilibrium temperature, $\mathbf{n}$ and $V$ are the unit normal to $\Gamma_{t}$ pointing into the liquid region and the normal velocity of the interface, respectively, and $\mathbf{T}(\boldsymbol{v}, p)=-p \mathbf{I}+2 v \rho \mathbf{D}(\boldsymbol{v})$ is the stress tensor. The notation $\boldsymbol{a}^{t}$ is used for the transposed vector of $\boldsymbol{a}$. In addition, we assume 
that the equilibrium pressure $p_{m}$ at the interface is given by

$$
p_{m}=-\frac{\rho \rho_{e} l}{\rho-\rho_{e}}\left(\ln T_{m}-\ln T_{c}\right),
$$

where $T_{c}$ is a constant, and, for convenience, instead of $T, T_{m}$, we take $T-T_{c}, T_{m}-T_{c}$ as unknowns and denote them by $T, T_{m}$. To complete the problem, we give the boundary condition on $\Sigma$,

$$
\left.\boldsymbol{v}\right|_{\Sigma}=\mathbf{0},\left.\quad T\right|_{\Sigma}=H
$$

and the initial condition

$$
\left.(\boldsymbol{v}, T)\right|_{t=0}=\left(\boldsymbol{v}_{0}, T_{0}\right) \quad \text { on } \bar{\Omega} \equiv \bar{\Omega}_{0} .
$$

When two phases are in equilibrium, a variation in pressure induces a corresponding change in temperature. This is an important consideration in technological applications. For example, the use of pressure as a factor in controlling crystal growth has been reported [8], [11], [19]. In order to take into account this pressure effect, we adopt the condition $(3)$ as a thermodynamic condition at the interface. This is achieved by integrating the equation

$$
\frac{d p_{m}}{d T_{m}}=-\frac{\rho \rho_{e} l}{\rho-\rho_{e}} \cdot \frac{1}{T_{m}},
$$

known as the Clapeyron-Clausius equation.

The Stefan problem is a mathematical model describing the process of liquid/solid phase transition. If the liquid region is assumed to be stagnant, this problem is formulated as a free boundary problem for the heat equation, in which the unknowns are the interface separating the liquid region and the solid region and the temperature distributions in both regions (see, e.g., [13], [15], [25]). Since the 1980's, more generalized Stefan problems, which describe the phase transition in flowing media, have been investigated in mathematics (see, e.g., [1], [2], [4], [5], [9]). However, in many cases, in the formulation of models, the melting point is given independently of the pressure, which means that the pressure effect mentioned above is neglected. The primary difference between the problem examined in the present paper and the models considered in earlier studies is that such a pressure effect is taken into account. This is a new problem, and as far as the authors know, there is no mathematically exact result.

In the present paper, we prove the unique (global-in-time) solvability of problem (1)-(5). The basic concept of the proof is based on a study by Shibata and Shimizu [18], who proved the existence of a global-in-time solution of the free boundary problem for the Navier-Stokes equations by iteration based on a maximal regularity result for the linearized problem on the time interval $(0, \infty)$.

The remainder of the present paper is organized as follows. In Section 2, we rewrite the problem as an initial boundary value problem defined in the initial domain and state the main result. Sections 3 and 4 are devoted to the investigation of the linear problem, which is arranged to define an iteration scheme to obtain the global solution to the nonlinear problem. In Section 3, we consider the resolvent problem, and, in Section 4, we prove the maximal regularity result. Finally in Section 5, we solve the nonlinear problem by successive approximation.

\section{Reduction of the problem and the main result}

The first step of the proof is to rewrite problems (1) through $(5)$ as an initial-boundary value problem defined in a domain with a fixed boundary. The transformation from Eulerian coordinates to Lagrangian coordinates was first introduced in order to solve the free boundary problems of the 
Navier-Stokes equations [23]. This method has an advantage in that no geometric restrictions on the interface are required, although the following kinematic condition is required. The interface consists of the same particles as those located on the interface at the initial time. Such a condition cannot be assumed in the phase transition problem, because mass transfer occurs at the interface. However, in the model considered herein, the following consideration enables the use of the transformation into the Lagrangian coordinates.

Let us define $\boldsymbol{v}^{\prime} \equiv\left(1-\rho_{e} / \rho\right)^{-1} \boldsymbol{v}$. Then we have

$$
\begin{aligned}
& \left\{\begin{array}{l}
\nabla \cdot \boldsymbol{v}^{\prime}=0, \quad \frac{\partial \boldsymbol{v}^{\prime}}{\partial t}+\left(1-\frac{\rho_{e}}{\rho}\right)\left(\boldsymbol{v}^{\prime} \cdot \nabla\right) \boldsymbol{v}^{\prime}-v \Delta \boldsymbol{v}^{\prime}+\left(\rho-\rho_{e}\right)^{-1} \nabla p=\mathbf{0}, \\
\frac{\partial T}{\partial t}+\left(1-\frac{\rho_{e}}{\rho}\right)\left(\boldsymbol{v}^{\prime} \cdot \nabla\right) T-\frac{\kappa}{\rho C_{p}} \Delta T \\
=\frac{2 v}{C_{p}}\left(1-\frac{\rho_{e}}{\rho}\right)^{2} \mathbf{D}\left(\boldsymbol{v}^{\prime}\right): \mathbf{D}\left(\boldsymbol{v}^{\prime}\right) \quad \text { in } \Omega_{t} \times\{t\}, t>0,
\end{array}\right. \\
& \left\{\begin{array}{l}
\boldsymbol{v}^{\prime} \cdot \mathbf{n}=V, \\
2 v \mathbf{D}\left(\boldsymbol{v}^{\prime}\right) \mathbf{n}-\left(\rho-\rho_{e}\right)^{-1} p \mathbf{n} \\
\quad=\frac{1}{\rho}\left[\boldsymbol{v}^{\prime}\left(\left(1-\frac{\rho_{e}}{\rho}\right) \boldsymbol{v}^{\prime}-V \mathbf{n}\right)^{t}\right] \mathbf{n}+\frac{\rho \rho_{e} l}{\left(\rho-\rho_{e}\right)^{2}}\left(\ln \left(T+T_{c}\right)-\ln T_{c}\right) \mathbf{n}, \\
l \rho_{e} V=-\kappa \nabla T \cdot \mathbf{n} \quad \text { on } \Gamma_{t} \times\{t\}, t>0, \\
\boldsymbol{v}^{\prime}=\mathbf{0}, \quad T=H \quad \text { on } \Sigma \times\{t\}, t>0,
\end{array}\right. \\
& \left.\boldsymbol{v}^{\prime}\right|_{t=0}=\left(1-\frac{\rho_{e}}{\rho}\right)^{-1} \boldsymbol{v}_{0},\left.\quad T\right|_{t=0}=T_{0} \quad \text { on } \bar{\Omega} .
\end{aligned}
$$

Note that, in the above problem, the kinematic condition, $V=\boldsymbol{v}^{\prime} \cdot \mathbf{n}$, is satisfied. Hence, by the transformation from the Eulerian coordinates $(x, t)$ to the Lagrangian coordinates $(\xi, t)$ defined by

$$
x=X_{\boldsymbol{u}}(\xi, t) \equiv \xi+\int_{0}^{t} \boldsymbol{u}(\xi, \tau) \mathrm{d} \tau,
$$

where $\boldsymbol{u}(\xi, t)$ is the velocity at time $t$ of the particle located at $\xi$ at $t=0$, we can rewrite this problem in the given cylindrical domain $\Omega_{\bar{t}}=\Omega \times(0, \bar{t})$ as follows:

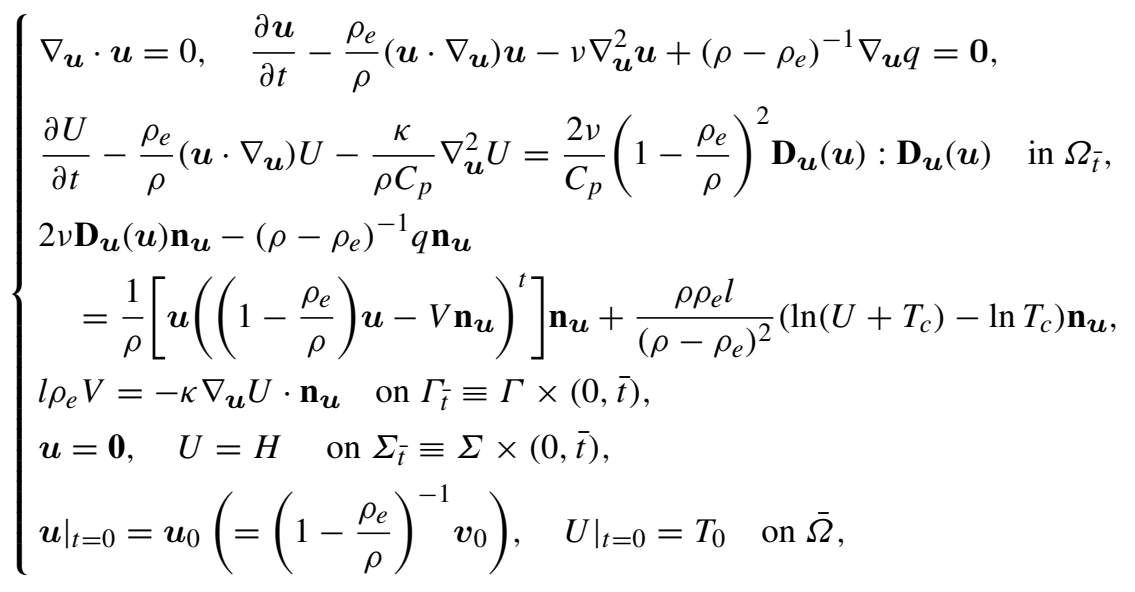


where $q=p \circ X_{\boldsymbol{u}}, U=T \circ X_{\boldsymbol{u}}, \nabla_{\boldsymbol{u}}=\left(\mathcal{J}^{-1}\right)^{t} \nabla \equiv \mathcal{J}^{*} \nabla, \mathbf{n}_{\boldsymbol{u}}=\mathbf{n} \circ X_{\boldsymbol{u}}$, and $\mathbf{D}_{\boldsymbol{u}}(\boldsymbol{u})$ is the tensor with elements $\frac{1}{2} \sum_{k=1}^{3}\left(a^{j k} \frac{\partial u_{i}}{\partial \xi_{k}}+a^{i k} \frac{\partial u_{j}}{\partial \xi_{k}}\right)(i, j=1,2,3)$. Here, we denote the Jacobian matrix of $X_{\boldsymbol{u}}$ by $\mathcal{J}$ and the $(i, j)$-element of $\mathcal{J}^{*}$ by $a^{i j}$.

Now, let us introduce the function spaces. Let $m \geqslant 0$, and let $1 \leqslant p \leqslant \infty$. In addition, let $\Omega \subset \mathbb{R}^{n}$. Here, $W_{p}^{m}(\Omega)$ denotes the space of functions defined on the domain $\Omega$ with the finite norm defined as follows:

If $m$ is an integer, then

$$
\|f\|_{p, \Omega}^{(m)} \equiv \sum_{|\alpha|=m}\left\|D^{\alpha} f\right\|_{p, \Omega}
$$

where

$$
\|f\|_{p, \Omega} \equiv\left(\int_{\Omega}|f(x)|^{p} \mathrm{~d} x\right)^{1 / p} \quad(1 \leqslant p<\infty), \quad\|f\|_{\infty, \Omega} \equiv \underset{x \in \Omega}{\operatorname{ess} \sup }|f(x)| .
$$

If $m$ is not an integer,

$$
\|f\|_{p, \Omega}^{(m)}=\|f\|_{p, \Omega}^{([m])}+[f]_{p, \Omega}^{(m)}
$$

where

$$
[f]_{p, \Omega}^{(m)}=\sum_{|\alpha|=[m]}\left(\int_{\Omega} \int_{\Omega} \frac{\left|D^{\alpha} f(x)-D^{\alpha} f(y)\right|^{p}}{|x-y|^{n+p(m-[m])}} \mathrm{d} x \mathrm{~d} y\right)^{1 / p} .
$$

Next, let $\Omega_{T} \equiv \Omega \times(0, T)$. Then $W_{p}^{m, l}\left(\Omega_{T}\right)$ denotes the space

$$
L_{p}\left(0, T ; W_{p}^{m}(\Omega)\right) \cap W_{p}^{l}\left(0, T ; L_{p}(\Omega)\right)
$$

with the norm

$$
\|f\|_{p, \Omega_{T}}^{(m, l)}=\|f\|_{p, \Omega_{T}}^{(m, 0)}+\|f\|_{p, \Omega_{T}}^{(0, l)}
$$

where

$$
\|f\|_{p, \Omega_{T}}^{(m, 0)} \equiv\left(\int_{0}^{T}\left(\|f(\cdot, t)\|_{p, \Omega}^{(m)}\right)^{p} \mathrm{~d} t\right)^{1 / p}, \quad\|f\|_{p, \Omega_{T}}^{(0, l)} \equiv\left(\int_{\Omega}\left(\|f(x, \cdot)\|_{p,(0, T)}^{(l)}\right)^{p} \mathrm{~d} x\right)^{1 / p} .
$$

For the same space defined on a cylinder $\Omega_{\infty} \equiv \Omega \times(0, \infty)$, we use the same notation as above, except that $\infty$ is used instead of $T$.

Finally, $W_{p}^{-1}(\Omega)=W_{0, \Gamma, p^{\prime}}^{1}(\Omega)^{*}$ denotes the dual space of $W_{0, \Gamma, p^{\prime}}^{1}(\Omega) \equiv\left\{\phi \in W_{p^{\prime}}^{1}(\Omega) \mid\right.$ $\left.\left.\phi\right|_{\Gamma}=0\right\}$, where $p^{\prime}=p /(p-1)$, with the norm

$$
\|f\|_{p, \Omega}^{(-1)}=\sup \left\{\left|\int_{\Omega} f \bar{\phi} \mathrm{d} x\right| \mid\|\nabla \phi\|_{p^{\prime}, \Omega}=1, \phi \in W_{0, \Gamma, p^{\prime}}^{1}(\Omega)\right\} .
$$

The following is the main result of the present paper.

THEOREM 2.1 Let $3<q<\infty$. Assume that $\boldsymbol{u}_{0} \in W_{q}^{2-2 / q}(\Omega), \quad T_{0} \in W_{q}^{2-2 / q}(\Omega), \quad H \in W_{q}^{2-1 / q, 1-1 / 2 q}\left(\Sigma_{\infty}\right), \quad \Gamma=\Gamma_{0} \in C^{2}, \quad \Sigma \in C^{2}$. 
Then there exists a constant $K$ such that if $\left\|\boldsymbol{u}_{0}\right\|_{q, \Omega}^{(2-2 / q)}+\left\|T_{0}\right\|_{q, \Omega}^{(2-2 / q)}+\|H\|_{q, \Sigma_{\infty}}^{(2-1 / q, 1-1 / 2 q)} \leqslant K^{2}$, then problem 8 with $\bar{t}=\infty$ has a unique solution $(\boldsymbol{u}, q, U) \in W_{q}^{2,1}\left(\Omega_{\infty}\right) \times W_{q}^{1,0}\left(\Omega_{\infty}\right) \times$ $W_{q}^{2,1}\left(\Omega_{\infty}\right)$ satisfying

$$
\left\|e^{\gamma t} \boldsymbol{u}\right\|_{q, \Omega_{\infty}}^{(2,1)}+\left\|e^{\gamma t} q\right\|_{q, \Omega_{\infty}}^{(1,0)}+\left\|e^{\gamma t} U\right\|_{q, \Omega_{\infty}}^{(2,1)} \leqslant K
$$

for some positive constant $\gamma$.

\section{Resolvent estimate}

In this section, we consider the following problem:

$$
\left\{\begin{array}{l}
\lambda \boldsymbol{u}-v \Delta \boldsymbol{u}+c_{0} \nabla q=\mathbf{f}, \quad \nabla \cdot \boldsymbol{u}=g, \\
\lambda U-\chi \Delta U=h \quad \text { in } \Omega, \\
2 v \Pi \mathbf{D}(\boldsymbol{u}) \mathbf{n}=\mathbf{F}\left(=\left(F_{1}, F_{2}\right)\right), \quad 2 \nu \mathbf{D}(\boldsymbol{u}) \mathbf{n} \cdot \mathbf{n}-c_{0} q-c_{1} U=F_{3}, \\
\nabla U \cdot \mathbf{n}+c_{2} \boldsymbol{u} \cdot \mathbf{n}=G \quad \text { on } \Gamma, \\
\boldsymbol{u}=\mathbf{0}, \quad U=H \quad \text { on } \Sigma .
\end{array}\right.
$$

Here, $\Pi$ is a projection operator on $\Gamma$ defined by $\Pi \mathbf{f}=\mathbf{f}-(\mathbf{f} \cdot \mathbf{n}) \mathbf{n}$.

The goal here is to prove the following theorem.

TheOREM 3.1 Let $1<q<\infty$ and $\pi / 3<\epsilon<\pi / 2$. Assume that

$$
\begin{gathered}
\mathbf{f} \in L_{q}(\Omega), \quad g \in W_{q}^{1}(\Omega) \cap W_{q}^{-1}(\Omega), \quad h \in L_{q}(\Omega), \quad \mathbf{F}=\left(F_{1}, F_{2}\right), F_{3} \in W_{q}^{1-1 / q}(\Gamma), \\
G \in W_{q}^{1-1 / q}(\Gamma), \quad H \in W_{q}^{2-1 / q}(\Sigma), \quad \Gamma \in C^{2}, \quad \Sigma \in C^{2} .
\end{gathered}
$$

Then, for every $\lambda \in \Sigma_{\epsilon} \cup\{0\}$, where $\Sigma_{\epsilon} \equiv\{\lambda \mid \lambda \neq 0,-\pi+\epsilon<\arg \lambda<\pi-\epsilon\}$, problem (9) has a unique solution $(\boldsymbol{u}, q, U) \in W_{q}^{2}(\Omega) \times W_{q}^{1}(\Omega) \times W_{q}^{2}(\Omega)$ satisfying

$$
\begin{aligned}
{[u]_{q, \Omega}^{(2)}+|\lambda| \| } & \boldsymbol{u}\left\|_{q, \Omega}+\right\| q\left\|_{q, \Omega}^{(1)}+[U]_{q, \Omega}^{(2)}+|\lambda|\right\| U \|_{q, \Omega} \\
\leqslant & C\left(\|\mathbf{f}\|_{q, \Omega}+[g]_{q, \Omega}^{(1)}+|\lambda|\|g\|_{q, \Omega}^{(-1)}+\|h\|_{q, \Omega}\right. \\
& +[\mathbf{F}]_{q, \Gamma}^{(1-1 / q)}+|\lambda|^{1 / 2-1 / 2 q}\|\mathbf{F}\|_{q, \Gamma}+\left[F_{3}\right]_{q, \Gamma}^{(1-1 / q)}+|\lambda|^{1 / 2-1 / 2 q}\left\|F_{3}\right\|_{q, \Gamma} \\
& \left.+[G]_{q, \Gamma}^{(1-1 / q)}+|\lambda|^{1 / 2-1 / 2 q}\|G\|_{q, \Gamma}+[H]_{q, \Sigma}^{(2-1 / q)}+|\lambda|^{1-1 / 2 q}\|H\|_{q, \Sigma}\right),
\end{aligned}
$$

where $C=C(q, \epsilon, \Omega)$ is a positive constant.

REMARK The constants $c_{0}, c_{1}$, and $c_{2}$ correspond to $\left(\rho-\rho_{e}\right)^{-1}, \rho \rho_{e} l /\left(\left(\rho-\rho_{e}\right)^{2} T_{c}\right)$, and $l \rho_{e} / \kappa$. Note that in both cases $\rho-\rho_{e}>0$ and $\rho-\rho_{e}<0$, we can assume that $c_{1} c_{2}$ is positive. This assumption is essential for the argument in this section and the following section.

First, we prove the existence of the weak solution. By introducing the new unknown functions $\boldsymbol{u}-\nabla \Phi$ and $U-\bar{U}$, where $\Phi, \bar{U}$ are the solutions of the problems

$$
\left\{\begin{array}{l}
\Delta \Phi=g \text { in } \Omega \\
\Phi=0 \quad \text { on } \Gamma, \quad \nabla \Phi \cdot \mathbf{n}=0 \quad \text { on } \Sigma
\end{array}\right.
$$




$$
\left\{\begin{array}{l}
\lambda \bar{U}-\chi \Delta \bar{U}=0 \quad \text { in } \Omega, \\
\nabla \bar{U} \cdot \mathbf{n}=0 \quad \text { on } \Gamma, \quad \bar{U}=H \quad \text { on } \Sigma,
\end{array}\right.
$$

we can reduce problem (9) to the problem with $g=0, H=0$. Hence, we assume $g=H=0$ in the following argument.

Through integration by parts, we have

$\lambda \int_{\Omega} \boldsymbol{u} \cdot \boldsymbol{v} \mathrm{d} x+2 v \int_{\Omega} \mathbf{D}(\boldsymbol{u}): \mathbf{D}(\boldsymbol{v}) \mathrm{d} x-\int_{\Gamma} c_{1} U \boldsymbol{v} \cdot \mathbf{n} \mathrm{d} \Gamma=\int_{\Omega} \mathbf{f} \cdot \boldsymbol{v} \mathrm{d} x+\int_{\Gamma}\left(\mathbf{F}, F_{3}\right) \cdot \boldsymbol{v} \mathrm{d} \Gamma$

and

$$
\lambda \int_{\Omega} U T \mathrm{~d} x+\chi \int_{\Omega} \nabla U \cdot \nabla T \mathrm{~d} x+\int_{\Gamma} c_{2} \chi T(\boldsymbol{u} \cdot \mathbf{n}) \mathrm{d} \Gamma=\int_{\Omega} h T \mathrm{~d} x+\int_{\Gamma} \chi G T \mathrm{~d} \Gamma .
$$

Multiplying (12) by $c_{1} /\left(c_{2} \chi\right)$ and adding to $[11$, we have

$$
\begin{aligned}
\lambda \int_{\Omega}\left(\boldsymbol{u} \cdot \boldsymbol{v}+\frac{c_{1}}{c_{2} \chi} U T\right) & \mathrm{d} x+2 v \int_{\Omega} \mathbf{D}(\boldsymbol{u}): \mathbf{D}(\boldsymbol{v}) \mathrm{d} x+\frac{c_{1}}{c_{2}} \int_{\Omega} \nabla U \cdot \nabla T \mathrm{~d} x-c_{1} \int_{\Gamma}(U \boldsymbol{v}-T \boldsymbol{u}) \cdot \mathbf{n} \mathrm{d} \Gamma \\
& =\int_{\Omega} \mathbf{f} \cdot \boldsymbol{v} \mathrm{d} x+\frac{c_{1}}{c_{2} \chi} \int_{\Omega} h T \mathrm{~d} x+\int_{\Gamma}\left(\mathbf{F}, F_{3}\right) \cdot \boldsymbol{v} \mathrm{d} \Gamma+\frac{c_{1}}{c_{2}} \int_{\Gamma} G T \mathrm{~d} \Gamma
\end{aligned}
$$

The left-hand side of this equality defines a bilinear form $B_{\lambda}[(\boldsymbol{u}, U),(\boldsymbol{v}, T)]$. Then the following condition is also satisfied:

$$
\left|B_{\lambda}[(\boldsymbol{u}, U),(\boldsymbol{u}, U)]\right| \geqslant C(\lambda)\left(\left(\|\boldsymbol{u}\|_{2, \Omega}^{(1)}\right)^{2}+\left(\|U\|_{2, \Omega}^{(1)}\right)^{2}\right)
$$

for arbitrary $(\boldsymbol{u}, U) \in W_{\sigma, 2}^{1}(\Omega) \times W_{0, \Sigma, 2}^{1}(\Omega)$, where $W_{\sigma, 2}^{1}(\Omega)$ and $W_{0, \Sigma, 2}^{1}(\Omega)$ are the function spaces defined as $W_{\sigma, 2}^{1}(\Omega)=\left\{\mathbf{f} \in W_{2}^{1}(\Omega)|\nabla \cdot \mathbf{f}=0, \mathbf{f} \cdot \mathbf{n}|_{\Sigma}=0\right\}$ and $W_{0, \Sigma, 2}^{1}(\Omega)=\left\{f \in W_{2}^{1}(\Omega) \mid\right.$ $\left.\left.f\right|_{\Sigma}=0\right\}$, respectively. Hence, the Lax-Milgram theorem implies the following theorem:

THEOREM 3.2 Let $W_{\sigma, 2}^{1}(\Omega)^{*}$ and $W_{0, \Sigma, 2}^{1}(\Omega)^{*}$ be the dual spaces of $W_{\sigma, 2}^{1}(\Omega)$ and $W_{0, \Sigma, 2}^{1}(\Omega)$, respectively. Then, for arbitrary $\lambda \in \Sigma_{\epsilon}, \mathbf{f} \in W_{\sigma, 2}^{1}(\Omega)^{*}, h \in W_{0, \Sigma, 2}^{1}(\Omega)^{*}, \mathbf{F}, F_{3}, G \in L_{2}(\Gamma)$, there exists a unique $(\boldsymbol{u}, U) \in W_{\sigma, 2}^{1}(\Omega) \times W_{0, \Sigma, 2}^{1}(\Omega)$ satisfying the equality 13 .

Now, let us consider the case of $\lambda=0$. Set $\mathbf{f}=\mathbf{0}, h=0, \mathbf{F}=\mathbf{0}$, and $F_{3}=G=0$. Then, from (13) we have

$$
[u]_{2, \Omega}^{(1)}+[U]_{2, \Omega}^{(1)} \leqslant C B_{0}[(u, U),(u, U)]^{1 / 2}=0 .
$$

In addition, using Korn's inequality and Poincaré's inequality, we have $\|\boldsymbol{u}\|_{2, \Omega}+\|U\|_{2, \Omega}=0$, and so it follows that $\boldsymbol{u}=\mathbf{0}$ and $U=0$. Thus, the conclusion of Theorem 3.2 holds for $\lambda=0$.

Now, we proceed to the estimation of the solution. Here, the proof is based on Schauder's method. The problem is reduced to the following whole space problem and half-space problems using a partition of unity:

$$
\left\{\begin{array}{l}
\lambda \boldsymbol{u}-v \Delta \boldsymbol{u}+c_{0} \nabla q=\mathbf{f}, \quad \nabla \cdot \boldsymbol{u}=g, \\
\lambda U-\chi \Delta U=h \quad \text { in } \mathbb{R}^{3},
\end{array}\right.
$$




$$
\left\{\begin{array}{l}
\lambda \boldsymbol{u}-v \Delta \boldsymbol{u}+c_{0} \nabla q=\mathbf{f}, \quad \nabla \cdot \boldsymbol{u}=g, \\
\lambda U-\chi \Delta U=h \quad \text { in } \mathbb{R}_{+}^{3} \equiv\left\{\left(z_{1}, z_{2}, z_{3}\right) \in \mathbb{R}^{3} \mid z_{3}>0\right\}, \\
\left.\boldsymbol{u}\right|_{z_{3}=0}=\mathbf{I},\left.\quad U\right|_{z_{3}=0}=H \quad \text { on } \mathbb{R}^{2},
\end{array}\right.
$$

and

$$
\left\{\begin{array}{l}
\lambda \boldsymbol{u}-v \Delta \boldsymbol{u}+c_{0} \nabla q=\mathbf{f}, \quad \nabla \cdot \boldsymbol{u}=g, \\
\lambda U-\chi \Delta U=h \quad \text { in } \mathbb{R}_{+}^{3}, \\
\left.v\left(\frac{\partial u_{j}}{\partial z_{3}}+\frac{\partial u_{3}}{\partial z_{j}}\right)\right|_{z_{3}=0}=F_{j} \quad(j=1,2), \\
2 v \frac{\partial u_{3}}{\partial z_{3}}-c_{0} q-\left.c_{1} U\right|_{z_{3}=0}=F_{3}, \quad \frac{\partial U}{\partial z_{3}}+\left.c_{2} u_{3}\right|_{z_{3}=0}=G \quad \text { on } \mathbb{R}^{2} .
\end{array}\right.
$$

Before stating the results, let us introduce the following function spaces:

- $\dot{W}_{q}^{-1}\left(\mathbb{R}^{3}\right)=\dot{W}_{q^{\prime}}^{1}\left(\mathbb{R}^{3}\right)^{*}$, where

$$
\dot{W}_{q^{\prime}}^{1}\left(\mathbb{R}^{3}\right)=\left\{f \in W_{q^{\prime}, l o c}^{1}\left(\mathbb{R}^{3}\right) \mid D f \in L_{q^{\prime}}\left(\mathbb{R}^{3}\right)\right\}, \quad q^{\prime}=\frac{q}{q-1} .
$$

- $\dot{W}_{q}^{-1}\left(\mathbb{R}_{+}^{3}\right)=\dot{W}_{q^{\prime}}^{1}\left(\mathbb{R}_{+}^{3}\right)^{*}$, where

$$
\dot{W}_{q^{\prime}}^{1}\left(\mathbb{R}_{+}^{3}\right)=\left\{f \in W_{q^{\prime}, \text { loc }}^{1}\left(\mathbb{R}_{+}^{3}\right) \mid D f \in L_{q^{\prime}}\left(\mathbb{R}_{+}^{3}\right)\right\}, \quad q^{\prime}=\frac{q}{q-1} .
$$

- $\dot{W}_{0, q}^{-1}\left(\mathbb{R}_{+}^{3}\right)=\dot{W}_{0, q^{\prime}}^{1}\left(\mathbb{R}_{+}^{3}\right)^{*}$, where

$$
\dot{W}_{0, q^{\prime}}^{1}\left(\mathbb{R}_{+}^{3}\right)=\left\{f \in W_{q^{\prime}, \text { loc }}^{1}\left(\mathbb{R}_{+}^{3}\right)\left|D f \in L_{q^{\prime}}\left(\mathbb{R}_{+}^{3}\right), f\right|_{z_{3}=0}=0\right\}, \quad q^{\prime}=\frac{q}{q-1} .
$$

For problems 15, , 16, and 17], we have the following results.

Theorem 3.3 Let $1<q<\infty$ and $0<\epsilon<\pi / 2$. Assume that

$$
\mathbf{f} \in L_{q}\left(\mathbb{R}^{3}\right), \quad g \in W_{q}^{1}\left(\mathbb{R}^{3}\right) \cap \dot{W}_{q}^{-1}\left(\mathbb{R}^{3}\right), \quad h \in L_{q}\left(\mathbb{R}^{3}\right),
$$

and that $g$ has a compact support. Then, for every $\lambda \in \Sigma_{\epsilon}$, problem (15) has a unique solution $(\boldsymbol{u}, \nabla q, U) \in W_{q}^{2}\left(\mathbb{R}^{3}\right) \times L_{q}\left(\mathbb{R}^{3}\right) \times W_{q}^{2}\left(\mathbb{R}^{3}\right)$ satisfying

$$
\begin{aligned}
{[u]_{q, \mathbb{R}^{3}}^{(2)}+|\lambda|\|\boldsymbol{u}\|_{q, \mathbb{R}^{3}}+\|\nabla q\|_{q, \mathbb{R}^{3}} } & +[U]_{q, \mathbb{R}^{3}}^{(2)}+|\lambda|\|U\|_{q, \mathbb{R}^{3}} \\
& \leqslant C\left(\|\mathbf{f}\|_{q, \mathbb{R}^{3}}+[g]_{q, \mathbb{R}^{3}}^{(1)}+|\lambda|\|g\|_{q, \mathbb{R}^{3}}^{(-1)}+\|h\|_{q, \mathbb{R}^{3}}\right),
\end{aligned}
$$

where $C=C(q, \epsilon)$ is a positive constant.

Theorem 3.4 Let $1<q<\infty$ and $0<\epsilon<\pi / 2$. Assume that

$\mathbf{f} \in L_{q}\left(\mathbb{R}_{+}^{3}\right), \quad g \in W_{q}^{1}\left(\mathbb{R}_{+}^{3}\right) \cap \dot{W}_{q}^{-1}\left(\mathbb{R}_{+}^{3}\right), \quad h \in L_{q}\left(\mathbb{R}_{+}^{3}\right), \quad \mathbf{I} \in W_{q}^{2-1 / q}\left(\mathbb{R}^{2}\right), \quad H \in W_{q}^{2-1 / q}\left(\mathbb{R}^{2}\right)$, 
and that $g$ has a compact support. Then, for every $\lambda \in \Sigma_{\epsilon}$, problem (16) has a unique solution $(\boldsymbol{u}, \nabla q, U) \in W_{q}^{2}\left(\mathbb{R}_{+}^{3}\right) \times L_{q}\left(\mathbb{R}_{+}^{3}\right) \times W_{q}^{2}\left(\mathbb{R}_{+}^{3}\right)$ satisfying

$$
\begin{aligned}
& {[\boldsymbol{u}]_{q, \mathbb{R}_{+}^{3}}^{(2)}+|\lambda|\|\boldsymbol{u}\|_{q, \mathbb{R}_{+}^{3}}+\|\nabla q\|_{q, \mathbb{R}_{+}^{3}}+[U]_{q, \mathbb{R}_{+}^{3}}^{(2)}+|\lambda|\|U\|_{q, \mathbb{R}_{+}^{3}} } \\
& \leqslant C\left(\|\mathbf{f}\|_{q, \mathbb{R}_{+}^{3}}+[g]_{q, \mathbb{R}_{+}^{3}}^{(1)}+|\lambda|\|g\|_{q, \mathbb{R}_{+}^{3}}^{(-1)}+\|h\|_{q, \mathbb{R}_{+}^{3}}\right. \\
&\left.+[\mathbf{I}]_{q, \mathbb{R}^{2}}^{(2-1 / q)}+|\lambda|^{1-1 / 2 q}\|\mathbf{I}\|_{q, \mathbb{R}^{2}}+[H]_{q, \mathbb{R}^{2}}^{(2-1 / q)}+|\lambda|^{1-1 / 2 q}\|H\|_{q, \mathbb{R}^{2}}\right)
\end{aligned}
$$

where $C=C(q, \epsilon)$ is a positive constant.

TheOREM 3.5 Let $1<q<\infty$ and $\pi / 3<\epsilon<\pi / 2$. Assume that

$$
\begin{gathered}
\mathbf{f} \in L_{q}\left(\mathbb{R}_{+}^{3}\right), \quad g \in W_{q}^{1}\left(\mathbb{R}_{+}^{3}\right) \cap \dot{W}_{0, q}^{-1}\left(\mathbb{R}_{+}^{3}\right), \quad h \in L_{q}\left(\mathbb{R}_{+}^{3}\right), \\
F_{1}, F_{2}, F_{3} \in W_{q}^{1-1 / q}\left(\mathbb{R}^{2}\right), \quad G \in W_{q}^{1-1 / q}\left(\mathbb{R}^{2}\right),
\end{gathered}
$$

and that $g$ has a compact support. Then, for every $\lambda \in \Sigma_{\epsilon}$, problem (17) has a unique solution $(\boldsymbol{u}, \nabla q, U) \in W_{q}^{2}\left(\mathbb{R}_{+}^{3}\right) \times L_{q}\left(\mathbb{R}_{+}^{3}\right) \times W_{q}^{2}\left(\mathbb{R}_{+}^{3}\right)$ satisfying

$$
\begin{aligned}
& {[\boldsymbol{u}]_{q, \mathbb{R}_{+}^{3}}^{(2)}+|\lambda|\|\boldsymbol{u}\|_{q, \mathbb{R}_{+}^{3}}+\|\nabla q\|_{q, \mathbb{R}_{+}^{3}}+[U]_{q, \mathbb{R}_{+}^{3}}^{(2)}+|\lambda|\|U\|_{q, \mathbb{R}_{+}^{3}}} \\
& \leqslant C\left(\|\mathbf{f}\|_{q, \mathbb{R}_{+}^{3}}+[g]_{q, \mathbb{R}_{+}^{3}}^{(1)}+|\lambda|\|g\|_{q, \mathbb{R}_{+}^{3}}^{(-1)}+\|h\|_{q, \mathbb{R}_{+}^{3}}\right. \\
& \left.\quad+\sum_{i=1}^{3}\left(\left[F_{i}\right]_{q, \mathbb{R}^{2}}^{(1-1 / q)}+|\lambda|^{1 / 2-1 / 2 q}\left\|F_{i}\right\|_{q, \mathbb{R}^{2}}\right)+[G]_{q, \mathbb{R}^{2}}^{(1-1 / q)}+|\lambda|^{1 / 2-1 / 2 q}\|G\|_{q, \mathbb{R}^{2}}\right)
\end{aligned}
$$

where $C=C(q, \epsilon)$ is a positive constant.

We next present the proof of Theorem 3.5. Theorems 3.3 and 3.4 can be proven in a similar, and simpler, manner.

Proof. In the proof, we use $C$ for various positive constants independent of $\lambda$. We seek the solution of problem 17 in the form

$$
\boldsymbol{u}=\mathbf{U}+\nabla V+\boldsymbol{w}, \quad q=-\frac{1}{c_{0}}(\lambda-\nu \Delta) V+\pi, \quad U=\tau+\theta,
$$

where $\mathbf{U}, V, \tau$ and $(\boldsymbol{w}, \pi, \theta)$ are solutions of the following problems:

$$
\begin{gathered}
\left\{\begin{array}{l}
\lambda \mathbf{U}-v \Delta \mathbf{U}=\mathbf{f} \quad \text { in } \mathbb{R}_{+}^{3}, \\
\left.\mathbf{U}\right|_{z_{3}=0}=\mathbf{0} \text { on } \mathbb{R}^{2},
\end{array}\right. \\
\left\{\begin{array}{l}
\Delta V=g-\nabla \cdot \mathbf{U} \equiv g^{\prime} \quad \text { in } \mathbb{R}_{+}^{3}, \\
\left.V\right|_{z_{3}=0}=0 \text { on } \mathbb{R}^{2},
\end{array}\right.
\end{gathered}
$$




$$
\begin{aligned}
& \left\{\begin{array}{l}
\lambda \tau-\chi \Delta \tau=h \quad \text { in } \mathbb{R}_{+}^{3}, \\
\left.\frac{\partial \tau}{\partial z_{3}}\right|_{z_{3}=0}=G-\left.c_{2}\left(\frac{\partial V}{\partial z_{3}}+U_{3}\right)\right|_{z_{3}=0} \equiv G^{\prime} \quad \text { on } \mathbb{R}^{2},
\end{array}\right. \\
& \left\{\begin{array}{l}
\lambda \boldsymbol{w}-v \Delta \boldsymbol{w}+c_{0} \nabla \pi= \\
\lambda \theta-\chi \Delta \theta=0 \quad \text { in } \mathbb{R}_{+}^{3} \\
\left.v\left(\frac{\partial w_{i}}{\partial z_{3}}+\frac{\partial w_{3}}{\partial z_{i}}\right)\right|_{z_{3}=0}
\end{array}\right. \\
& =F_{i}-\left.v \frac{\partial}{\partial z_{3}}\left(U_{i}+\frac{\partial V}{\partial z_{i}}\right)\right|_{z_{3}=0}-\left.v \frac{\partial}{\partial z_{i}}\left(U_{3}+\frac{\partial V}{\partial z_{3}}\right)\right|_{z_{3}=0} \quad(i=1,2), \\
& 2 v \frac{\partial w_{3}}{\partial z_{3}}-c_{0} \pi-\left.c_{1} \theta\right|_{z_{3}=0} \\
& =F_{3}-\left.2 v \frac{\partial}{\partial z_{3}}\left(U_{3}+\frac{\partial V}{\partial z_{3}}\right)\right|_{z_{3}=0}-\left.(\lambda V-v \Delta V)\right|_{z_{3}=0}+\left.c_{1} \tau\right|_{z_{3}=0}, \\
& c_{2} w_{3}+\left.\frac{\partial \theta}{\partial z_{3}}\right|_{z_{3}=0}=0 \quad \text { on } \mathbb{R}^{2} \text {. }
\end{aligned}
$$

For problems 21, 22, and 23, we have the following estimates:

$$
\begin{aligned}
|\lambda|\|\mathbf{U}\|_{q, \mathbb{R}_{+}^{3}}+[\mathbf{U}]_{q, \mathbb{R}_{+}^{3}}^{(2)} & \leqslant C\|\mathbf{f}\|_{q, \mathbb{R}_{+}^{3},} \\
|\lambda|\|\nabla V\|_{q, \mathbb{R}_{+}^{3}}+[\nabla V]_{q, \mathbb{R}_{+}^{3}}^{(2)} & \leqslant C\left(\left[g^{\prime}\right]_{q, \mathbb{R}_{+}^{3}}^{(1)}+|\lambda|\left\|g^{\prime}\right\|_{q, \mathbb{R}_{+}^{3}}^{(-1)}\right), \\
|\lambda|\|\tau\|_{q, \mathbb{R}_{+}^{3}}+[\tau]_{q, \mathbb{R}_{+}^{3}}^{(2)} & \leqslant C\left(\|h\|_{q, \mathbb{R}_{+}^{3}}+\left[G^{\prime}\right]_{q, \mathbb{R}^{2}}^{(1-1 / q)}+|\lambda|^{1 / 2-1 / 2 q}\left\|G^{\prime}\right\|_{q, \mathbb{R}^{2}}\right) .
\end{aligned}
$$

Next, let us prove the solvability of problem (24). To simplify the notation, we denote the members on the right-hand side again by $F_{1}, F_{2}$, and $F_{3}$. By applying the Fourier transformation

$$
\mathcal{F}(f)\left(\xi^{\prime}, z_{3}\right) \equiv \tilde{f}\left(\xi^{\prime}, z_{3}\right) \equiv \frac{1}{2 \pi} \int_{\mathbb{R}^{2}} e^{-i z^{\prime} \cdot \xi^{\prime}} f\left(z^{\prime}, z_{3}\right) \mathrm{d} z^{\prime}
$$

to 24, we obtain the following system of ordinary differential equations:

$$
\left\{\begin{array}{l}
\left(\lambda+v\left|\xi^{\prime}\right|^{2}\right) \tilde{w}_{1}-v \frac{\mathrm{d}^{2} \tilde{w}_{1}}{\mathrm{~d} z_{3}^{2}}+c_{0} i \xi_{1} \tilde{\pi}=0 \\
\left(\lambda+v|\xi|^{2}\right) \tilde{w}_{2}-v \frac{\mathrm{d}^{2} \tilde{w}_{2}}{\mathrm{~d} z_{3}^{2}}+c_{0} i \xi_{2} \tilde{\pi}=0 \\
\left(\lambda+v\left|\xi^{\prime}\right|^{2}\right) \tilde{w}_{3}-v \frac{\mathrm{d}^{2} \tilde{w}_{3}}{\mathrm{~d} z_{3}^{2}}+c_{0} \frac{\mathrm{d} \tilde{\pi}}{\mathrm{d} z_{3}}=0 \\
i \xi_{1} \tilde{w}_{1}+i \xi_{2} \tilde{w}_{2}+\frac{\mathrm{d} \tilde{w}_{3}}{\mathrm{~d} z_{3}}=0 \\
\left(\lambda+\chi\left|\xi^{\prime}\right|^{2}\right) \tilde{\theta}-\chi \frac{\mathrm{d}^{2} \tilde{\theta}}{\mathrm{d} z_{3}^{2}}=0
\end{array}\right.
$$


with boundary conditions

$$
\left\{\begin{array}{l}
\left.v\left(\frac{\partial \tilde{w}_{1}}{\partial z_{3}}+i \xi_{1} \tilde{w}_{3}\right)\right|_{z_{3}=0}=\tilde{F}_{1} \\
\left.v\left(\frac{\partial \tilde{w}_{2}}{\partial z_{3}}+i \xi_{2} \tilde{w}_{3}\right)\right|_{z_{3}=0}=\tilde{F}_{2} \\
2 v \frac{\partial \tilde{w}_{3}}{\partial z_{3}}-c_{0} \tilde{\pi}-\left.c_{1} \tilde{\theta}\right|_{z_{3}=0}=\tilde{F}_{3} \\
\frac{\partial \tilde{\theta}}{\partial z_{3}}+\left.c_{2} \tilde{w}_{3}\right|_{z_{3}=0}=0, \\
\tilde{w}_{i}, \tilde{\pi}, \tilde{\theta} \rightarrow 0 \quad\left(z_{3} \rightarrow \infty\right) .
\end{array}\right.
$$

Solving the above equations, we obtain

$$
\left\{\begin{array}{l}
\tilde{\theta}=-\frac{c_{1} c_{2}\left|\xi^{\prime}\right|\left(r+\left|\xi^{\prime}\right|\right) \tilde{F}_{3}+c_{1} c_{2}\left(r-\left|\xi^{\prime}\right|\right)\left(i \xi_{1} \tilde{F}_{1}+i \xi_{2} \tilde{F}_{2}\right)}{\nu\left(R Q+2 c_{1} c_{2}\left|\xi^{\prime}\right|\left(r+\left|\xi^{\prime}\right|\right)\right)} e^{-R z_{3}} \equiv \sum_{j=1}^{3} \tilde{G}_{j} e^{-R z_{3}} \tilde{F}_{j}, \\
\tilde{w}_{i}=\tilde{W}_{i}-\frac{c_{1} i \xi_{i} r\left(r-\left|\xi^{\prime}\right|\right)}{\nu Q} \sum_{j=1}^{3} \tilde{G}_{j} e^{-r z_{3}} \tilde{F}_{j}+\frac{c_{1} i \xi_{i}\left(r^{2}+\left|\xi^{\prime}\right|^{2}\right)}{\nu Q} \sum_{j=1}^{3} \tilde{G}_{j} e_{1}\left(z_{3}\right) \tilde{F}_{j}, \\
\tilde{w}_{3}=\tilde{W}_{3}+\frac{c_{1}\left|\xi^{\prime}\right|\left(r+\left|\xi^{\prime}\right|\right)}{\nu Q} \sum_{j=1}^{3} \tilde{G}_{j} e^{-r z_{3}} \tilde{F}_{j}-\frac{c_{1}\left|\xi^{\prime}\right|\left(r^{2}+\left|\xi^{\prime}\right|^{2}\right)}{\nu Q} \sum_{j=1}^{3} \tilde{G}_{j} e_{1}\left(z_{3}\right) \tilde{F}_{j}, \\
\tilde{\pi}=\tilde{P}+\frac{c_{1}\left(r+\left|\xi^{\prime}\right|\right)\left(r^{2}+\left|\xi^{\prime}\right|^{2}\right)}{c_{0} Q} \sum_{j=1}^{3} \tilde{G}_{j} e^{-\left|\xi^{\prime}\right| z_{3}} \tilde{F}_{j} .
\end{array}\right.
$$

Here,

$$
\left\{\begin{aligned}
\tilde{W}_{i}= & -\frac{\tilde{F}_{i}}{\nu r} e^{-r z_{3}}-i \xi_{i}\left(\frac{\left(3 r-\left|\xi^{\prime}\right|\right)\left(i \xi_{1} \tilde{F}_{1}+i \xi_{2} \tilde{F}_{2}\right)-r\left(r-\left|\xi^{\prime}\right|\right) \tilde{F}_{3}}{\nu r Q}\right) e^{-r z_{3}} \\
& +i \xi_{i}\left(\frac{2 r\left(i \xi_{1} \tilde{F}_{1}+i \xi_{2} \tilde{F}_{2}\right)-\left(r^{2}+\left|\xi^{\prime}\right|^{2}\right) \tilde{F}_{3}}{\nu Q}\right) e_{1}\left(z_{3}\right), \\
\tilde{W}_{3}= & -\frac{\left(r-\left|\xi^{\prime}\right|\right)\left(i \xi_{1} \tilde{F}_{1}+i \xi_{2} \tilde{F}_{2}\right)+\left|\xi^{\prime}\right|\left(r+\left|\xi^{\prime}\right|\right) \tilde{F}_{3}}{\nu Q} e^{-r z_{3}} \\
& -\frac{2\left|\xi^{\prime}\right| r\left(i \xi_{1} \tilde{F}_{1}+i \xi_{2} \tilde{F}_{2}\right)-\left|\xi^{\prime}\right|\left(r^{2}+\left|\xi^{\prime}\right|^{2}\right) \tilde{F}_{3}}{\nu Q} e_{1}\left(z_{3}\right), \\
\tilde{P}= & \frac{2\left(r+\left|\xi^{\prime}\right|\right) r\left(i \xi_{1} \tilde{F}_{1}+i \xi_{2} \tilde{F}_{2}\right)-\left(r+\left|\xi^{\prime}\right|\right)\left(r^{2}+\left|\xi^{\prime}\right|^{2}\right) \tilde{F}_{3}}{c_{0} Q} e^{-\left|\xi^{\prime}\right| z_{3}}
\end{aligned}\right.
$$

where

$$
\begin{aligned}
r=\sqrt{\frac{\lambda}{v}+\left|\xi^{\prime}\right|^{2}}, \quad R & =\sqrt{\frac{\lambda}{\chi}+\left|\xi^{\prime}\right|^{2}}, \quad e_{1}\left(z_{3}\right)=\frac{e^{-r z_{3}}-e^{-\left|\xi^{\prime}\right| z_{3}}}{r-\left|\xi^{\prime}\right|} \\
Q\left(\lambda,\left|\xi^{\prime}\right|\right) & =r^{3}+\left|\xi^{\prime}\right| r^{2}+3 r\left|\xi^{\prime}\right|^{2}-\left|\xi^{\prime}\right|^{3}
\end{aligned}
$$


For $W_{i}$ and $P$, the following estimate has been obtained in [17]:

$$
\sum_{i=1}^{3}\left(\left[W_{i}\right]_{q, \mathbb{R}_{+}^{3}}^{(2)}+|\lambda|\left\|W_{i}\right\|_{q, \mathbb{R}_{+}^{3}}\right)+\|\nabla P\|_{q, \mathbb{R}_{+}^{3}} \leqslant C \sum_{j=1}^{3}\left(\left[F_{j}\right]_{q, \mathbb{R}^{2}}^{(1-1 / q)}+|\lambda|^{1-1 / q}\left\|F_{j}\right\|_{q, \mathbb{R}^{2}}\right) .
$$

Let us estimate the remaining terms. We first prove that

$$
[\theta]_{q, \mathbb{R}_{+}^{3}}^{(2)}+|\lambda|\|\theta\|_{q, \mathbb{R}_{+}^{3}} \leqslant C \sum_{j=1}^{3}\left(\left[F_{j}\right]_{q, \mathbb{R}^{2}}^{(1-1 / q)}+|\lambda|^{1-1 / q}\left\|F_{j}\right\|_{q, \mathbb{R}^{2}}\right) .
$$

The following lemmas are used to derive this estimate.

LEMMA 3.1 ([17]) If $|\arg \lambda| \leqslant \pi-\epsilon$, then

$$
\left|Q\left(\lambda,\left|\xi^{\prime}\right|\right)\right| \geqslant C(\epsilon)\left(|r|^{2}+\left|\xi^{\prime}\right|^{2}\right)^{3 / 2}
$$

where $C(\epsilon)$ is a positive constant.

For $\lambda$ satisfying $|\arg \lambda|<2 \pi / 3$, we have $\left|\arg (R Q)-\arg \left(\left|\xi^{\prime}\right|\left(r+\left|\xi^{\prime}\right|\right)\right)\right|<\pi$. This implies the following lemma.

LEMMA 3.2 If $|\arg \lambda| \leqslant 2 \pi / 3-\epsilon$, then

$$
\left|R Q+2 c_{1} c_{2}\right| \xi^{\prime}\left|\left(r+\left|\xi^{\prime}\right|\right)\right| \geqslant C(\epsilon)\left(|R||Q|+\left|\xi^{\prime}\right|\left(|r|+\left|\xi^{\prime}\right|\right)\right),
$$

where $C(\epsilon)$ is a positive constant.

We set

$$
\begin{aligned}
& \theta_{j} \equiv-\int_{\mathbb{R}^{2}} e^{i z^{\prime} \cdot \xi^{\prime}} \frac{c_{1} c_{2}\left(r-\left|\xi^{\prime}\right|\right) i \xi_{i}}{v\left(R Q+2 c_{1} c_{2}\left|\xi^{\prime}\right|\left(r+\left|\xi^{\prime}\right|\right)\right)} e^{-R z_{3}} \tilde{F}_{j}\left(\xi^{\prime}\right) \mathrm{d} \xi^{\prime} \quad(j=1,2), \\
& \theta_{3} \equiv-\int_{\mathbb{R}^{2}} e^{i z^{\prime} \cdot \xi^{\prime}} \frac{c_{1} c_{2}\left|\xi^{\prime}\right|\left(r+\left|\xi^{\prime}\right|\right)}{v\left(R Q+2 c_{1} c_{2}\left|\xi^{\prime}\right|\left(r+\left|\xi^{\prime}\right|\right)\right)} e^{-R z_{3}} \tilde{F}_{3}\left(\xi^{\prime}\right) \mathrm{d} \xi^{\prime} .
\end{aligned}
$$

We estimate only $\theta_{3}$, because $\theta_{1}$ and $\theta_{2}$ can be estimated in the same manner. Since

$$
\int_{\mathbb{R}^{2}}\left[\int_{\mathbb{R}^{2}} e^{i z^{\prime} \cdot \xi^{\prime}} \frac{c_{1} c_{2}\left|\xi^{\prime}\right|\left(r+\left|\xi^{\prime}\right|\right)}{v\left(R Q+2 c_{1} c_{2}\left|\xi^{\prime}\right|\left(r+\left|\xi^{\prime}\right|\right)\right)} e^{-R z_{3}} \mathrm{~d} \xi^{\prime}\right] \mathrm{d} z^{\prime}=0
$$

we can write

$$
\theta_{3}=-\int_{\mathbb{R}^{2}}\left(\int_{\mathbb{R}^{2}} e^{i \zeta^{\prime} \cdot \xi^{\prime}} \frac{c_{1} c_{2}\left|\xi^{\prime}\right|\left(r+\left|\xi^{\prime}\right|\right)}{v\left(R Q+2 c_{1} c_{2}\left|\xi^{\prime}\right|\left(r+\left|\xi^{\prime}\right|\right)\right)} e^{-R z_{3}} \mathrm{~d} \xi^{\prime}\right)\left(F_{3}\left(z^{\prime}-\zeta^{\prime}\right)-F_{3}\left(z^{\prime}\right)\right) \mathrm{d} \zeta^{\prime} .
$$

Set

$$
I \equiv \sum_{k=0}^{2} \int_{\mathbb{R}^{2}} D_{\zeta^{\prime}}^{k}\left(e^{i \zeta^{\prime} \cdot \xi^{\prime}} \frac{c_{1} c_{2}\left|\xi^{\prime}\right|\left(r+\left|\xi^{\prime}\right|\right)}{v\left(R Q+2 c_{1} c_{2}\left|\xi^{\prime}\right|\left(r+\left|\xi^{\prime}\right|\right)\right)}\right) D_{z_{3}}^{2-k} e^{-R z_{3}} \mathrm{~d} \xi^{\prime}
$$


Let us choose a rotation of the coordinates such that $\mathcal{R} \zeta^{\prime}=\left(\left|\zeta^{\prime}\right|, 0\right)$, and let us perform the change of variables $\xi^{\prime}=|\lambda|^{1 / 2} \mathcal{R} \eta^{\prime}$ and $\zeta^{\prime}=|\lambda|^{-1 / 2} Z^{\prime}$. Then, by shifting the path of integration for $\alpha$ to the contour $s+i \delta(|\beta|+|s|), s \in \mathbb{R}, \delta>0$, where $(\alpha, \beta)=\mathcal{R} \eta^{\prime}, I$ is estimated as follows:

$$
|I| \leqslant C \sum_{k=0}^{2} \int_{0}^{\infty} \int_{0}^{\infty} e^{-\left|Z^{\prime}\right|(\beta+s)} \frac{|\lambda|^{2}\left|\mathcal{R} \eta^{\prime}\right|^{k+1}\left(r+\left|\mathcal{R} \eta^{\prime}\right|\right)|R|^{2-k}}{|\lambda|^{2}|R||Q|+|\lambda|\left|\mathcal{R} \eta^{\prime}\right|\left(|r|+\left|\mathcal{R} \eta^{\prime}\right|\right) \mid} e^{-R z_{3}}|\lambda| \mathrm{d} \beta \mathrm{d} s \equiv \sum_{k=0}^{2} I_{k} .
$$

In addition, let us introduce the new variable $\rho=\sqrt{\beta^{2}+s^{2}}$. Then we have

$$
\begin{aligned}
\left|I_{k}\right| & \leqslant C \int_{0}^{\infty} e^{-c\left|Z^{\prime}\right| \rho} \frac{|\lambda|^{3} \rho^{1+k}(1+\rho)^{3-k}}{|\lambda|^{2}(1+\rho)^{4}+|\lambda| \rho(1+\rho)} e^{-c(1+\rho)|\lambda|^{1 / 2} z_{3}} \rho \mathrm{d} \rho \\
& \leqslant C \int_{0}^{\infty} e^{-c\left|Z^{\prime}\right| \rho} \frac{|\lambda|^{3} \rho^{1+k}(1+\rho)^{3-k}}{|\lambda|^{3 / 2}(1+\rho)^{2} \rho^{1 / 2}(1+\rho)^{1 / 2}} e^{-c(1+\rho)|\lambda|^{1 / 2} z_{3}} \rho \mathrm{d} \rho \\
& \leqslant C \int_{0}^{\infty} e^{-c\left(\left|Z^{\prime}\right|+|\lambda|^{1 / 2} z_{3}\right) \rho}|\lambda|^{3 / 2} \rho^{2} \mathrm{~d} \rho \leqslant C \frac{|\lambda|^{3 / 2}}{\left(\left|Z^{\prime}\right|+|\lambda|^{1 / 2} z_{3}\right)^{3}} \leqslant \frac{C}{\left(\left|\zeta^{\prime}\right|^{2}+z_{3}^{2}\right)^{3 / 2}} .
\end{aligned}
$$

Using this estimate, we obtain

$$
\begin{aligned}
\left\|D_{z}^{2} \theta_{3}\right\|_{z^{\prime}, q} \leqslant & \int_{\mathbb{R}^{2}} \frac{1}{\left(\left|\zeta^{\prime}\right|^{2}+z_{3}^{2}\right)^{3 / 2}}\left\|F_{3}\left(z^{\prime}-\zeta^{\prime}\right)-F_{3}\left(z^{\prime}\right)\right\|_{z^{\prime}, q} \mathrm{~d} \zeta^{\prime} \\
& \leqslant\left(\int_{\mathbb{R}^{2}} \frac{\left\|F_{3}\left(z^{\prime}-\zeta^{\prime}\right)-F_{3}\left(z^{\prime}\right)\right\|_{z^{\prime}, q}^{q}}{\left(\left|\zeta^{\prime}\right|^{2}+z_{3}^{2}\right)^{3 / 4+q / 2}}\right)^{1 / q}\left(\int_{\mathbb{R}^{2}} \frac{\mathrm{d} \zeta^{\prime}}{\left(\left|\zeta^{\prime}\right|^{2}+z_{3}^{2}\right)^{1+q^{\prime} / 4 q}}\right)^{1 / q^{\prime}} \\
& \leqslant \frac{1}{z_{3}^{1 / 2 q}}\left(\int_{\mathbb{R}^{2}} \frac{\left\|F_{3}\left(z^{\prime}-\zeta^{\prime}\right)-F_{3}\left(z^{\prime}\right)\right\|_{z^{\prime}, q}^{q}}{\left(\left|\zeta^{\prime}\right|^{2}+z_{3}^{2}\right)^{3 / 4+q / 2}} \mathrm{~d} \zeta^{\prime}\right)^{1 / q}
\end{aligned}
$$

Thus,

$$
\begin{aligned}
\left\|D_{z}^{2} \theta_{3}\right\|_{q, \mathbb{R}_{+}^{3}} & \leqslant\left(\int_{\mathbb{R}^{2}}\left\|F_{3}\left(z^{\prime}-\zeta^{\prime}\right)-F_{3}\left(z^{\prime}\right)\right\|_{z^{\prime}, q}^{q} \mathrm{~d} \zeta^{\prime} \int_{0}^{\infty} \frac{d z_{3}}{z_{3}^{1 / 2}\left(\left|\zeta^{\prime}\right|^{2}+z_{3}^{2}\right)^{3 / 4+q / 2}}\right)^{1 / q} \\
& \leqslant C\left[F_{3}\right]_{q, \mathbb{R}^{2}}^{(1-1 / q)}
\end{aligned}
$$

On the other hand, we have

$$
\begin{aligned}
|\lambda|\|I\|_{1, \zeta^{\prime}, \mathbb{R}^{2}} & \leqslant C|\lambda| e^{-c|\lambda|^{1 / 2} z_{3}} \int_{\mathbb{R}^{2}} e^{-c\left|Z^{\prime}\right| \rho}|\lambda|^{-1} d Z^{\prime} \int_{0}^{\infty} \frac{|\lambda|^{2} \rho^{2}(1+\rho)}{|\lambda|^{3 / 2}(1+\rho)^{2} \rho^{1 / 2}(1+\rho)^{1 / 2}} \mathrm{~d} \rho \\
& \leqslant C|\lambda|^{\frac{1}{2}(1-1 / q)}|\lambda|^{1 / 2 q} e^{-|\lambda|^{1 / 2} z_{3}},
\end{aligned}
$$

which implies

$$
|\lambda|\left\|\theta_{3}\right\|_{q, \mathbb{R}_{+}^{2}} \leqslant C|\lambda|^{\frac{1}{2}(1-1 / q)}\left\|F_{3}\right\|_{q, \mathbb{R}^{2}} .
$$

Thus, we have the estimate given in (33).

Next, we estimate the remaining terms in 30 . We set

$$
K_{j, k}=\mathcal{F}^{-1}\left(-\frac{c_{1} i \xi_{j} r\left(r-\left|\xi^{\prime}\right|\right)}{\nu Q} \tilde{G}_{k} e^{-r z_{3}}\right), \quad L_{j, k}=\mathcal{F}^{-1}\left(\frac{c_{1} i \xi_{j}\left(r^{2}+\left|\xi^{\prime}\right|^{2}\right)}{\nu Q} \tilde{G}_{k} e_{1}\left(z_{3}\right)\right),
$$




$$
\begin{aligned}
K_{3, k} & =\mathcal{F}^{-1}\left(\frac{c_{1}\left|\xi^{\prime}\right|\left(r+\left|\xi^{\prime}\right|\right)}{\nu Q} \tilde{G}_{k} e^{-r z_{3}}\right), \quad L_{3, k}=\mathcal{F}^{-1}\left(-\frac{c_{1}\left|\xi^{\prime}\right|\left(r^{2}+\left|\xi^{\prime}\right|^{2}\right)}{\nu Q} \tilde{G}_{k} e_{1}\left(z_{3}\right)\right), \\
M_{k} & =\mathcal{F}^{-1}\left(\frac{c_{1}\left(r+\left|\xi^{\prime}\right|\right)\left(r^{2}+\left|\xi^{\prime}\right|^{2}\right)}{c_{0} Q} \tilde{G}_{k} e^{-\left|\xi^{\prime}\right| z_{3}}\right) \quad(j=1,2, k=1,2,3),
\end{aligned}
$$

where $\mathcal{F}^{-1}(f)$ denotes the inverse Fourier transformation

$$
\mathcal{F}^{-1}(f)\left(z^{\prime}, z_{3}\right) \equiv \frac{1}{2 \pi} \int_{\mathbb{R}^{2}} e^{i z^{\prime} \cdot \xi^{\prime}} f\left(\xi^{\prime}, z_{3}\right) \mathrm{d} \xi^{\prime}
$$

We then set

$$
\mathcal{W}_{i} \equiv \sum_{j=1}^{3}\left(K_{i, j} * F_{j}+L_{i, j} * F_{j}\right), \quad \mathcal{P} \equiv \sum_{j=1}^{3} M_{j} * F_{j},
$$

where $f * g$ denotes the convolution $\int_{\mathbb{R}^{2}} f\left(z^{\prime}-\zeta^{\prime}, z_{3}\right) g\left(\zeta^{\prime}\right) \mathrm{d} \zeta^{\prime}$.

Using the inequality $\left|R Q+2 c_{2} c_{3}\right| \xi^{\prime}\left|\left(r+\left|\xi^{\prime}\right|\right)\right| \geqslant C\left|\xi^{\prime}\right||r+| \xi^{\prime}||$, we have

$$
|\lambda|\left|K_{i, j}\right|+\left|D_{z}^{2} K_{i, j}\right|,\left|D_{z} M_{j}\right| \leqslant \frac{C}{\left(\left|z^{\prime}\right|^{2}+z_{3}^{2}\right)^{3 / 2}} .
$$

On the other hand, using the inequalities

$$
\left\{\begin{array}{l}
\left|e_{1}\left(z_{3}\right)\right|=e^{-\left|\xi^{\prime}\right| z_{3}}\left|\frac{1-e^{-\left(r-\left|\xi^{\prime}\right|\right) z_{3}}}{r-\left|\xi^{\prime}\right|}\right| \leqslant C e^{-\left|\xi^{\prime}\right| z_{3}}, \\
\left|R Q+2 c_{2} c_{3}\right| \xi^{\prime}\left|\left(r+\left|\xi^{\prime}\right|\right)\right| \geqslant C|R Q|^{1 / 2}\left|\xi^{\prime}\right|^{1 / 2}|r+| \xi^{\prime}||^{1 / 2}
\end{array}\right.
$$

we have the following estimate:

$$
|\lambda|\left|L_{i, j}\right|+\left|D_{z}^{2} L_{i, j}\right| \leqslant \frac{C}{\left(\left|z^{\prime}\right|^{2}+z_{3}^{2}\right)^{3 / 2}}
$$

which is derived in the same manner as (34). These inequalities imply

$$
\sum_{i=1}^{3}\left(|\lambda|\left\|\mathcal{W}_{i}\right\|_{q, \mathbb{R}_{+}^{3}}+\left[W_{i}\right]_{q, \mathbb{R}_{+}^{3}}^{(2)}\right) \leqslant C \sum_{j=1}^{3}\left[F_{j}\right]_{q, \mathbb{R}^{2}}^{(1-1 / q)}
$$

and

$$
\|\nabla \mathcal{P}\|_{q, \mathbb{R}_{+}^{3}} \leqslant C \sum_{j=1}^{3}\left[F_{j}\right]_{q, \mathbb{R}^{2}}^{(1-1 / q)} .
$$

Thus, we have completed the proof of Theorem 3.5.

Next, we derive an a priori estimate of the solution of problem (9). Let $x_{0}$ be an arbitrary point on $\Gamma$. Let us denote the neighborhood $\left\{x|| x-x_{0} \mid \leqslant \delta\right\}$ of $x_{0}$ by $B_{\delta}\left(x_{0}\right)$. Let $\phi \in C_{0}^{\infty}\left(\mathbb{R}^{3}\right)$ be such that $\phi(x)=1(|x|<1 / 2), \phi(x)=0(|x|>1)$. With no loss of generality, we may assume that $x_{0}$ 
is the origin and that the $x_{3}$ axis is directed along the normal to $\Gamma$ at $x_{0}$. Let us introduce the new coordinate $z$ defined by

$$
z^{\prime}=x^{\prime}, \quad z_{3}=x_{3}-F\left(x^{\prime}\right)
$$

where $F$ is a function defining the surface in the neighborhood of $x_{0}=0$ by $x_{3}=F\left(x^{\prime}\right)$. In addition, let us denote $\boldsymbol{u}(\mathcal{U} z), q(\mathcal{U} z), U(\mathcal{U} z)$ by $\overline{\boldsymbol{u}}, \bar{q}, \bar{U}$, where $\mathcal{U}$ represents the transformation from $x$ to $z$ defined above, and let us denote $\overline{\boldsymbol{u}} \phi_{\delta}(z), \bar{q} \phi_{\delta}(z), \bar{U} \phi_{\delta}(z)$ by $\tilde{\boldsymbol{u}}, \tilde{q}, \tilde{U}$, where $\phi_{\delta}(z)=\phi(z / \delta)$. Then $\tilde{\boldsymbol{u}}, \tilde{q}$, and $\tilde{U}$ satisfy

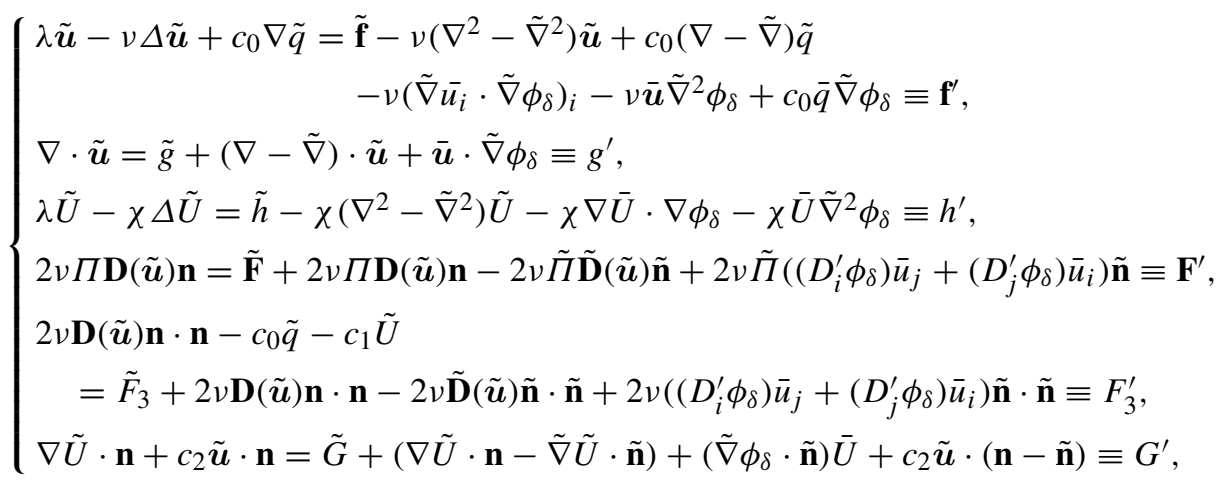

where

$$
\left\{\begin{array}{l}
\tilde{\nabla}=\left(D_{1}^{\prime}, D_{2}^{\prime}, D_{3}^{\prime}\right)=\left(\frac{\partial}{\partial z_{1}}-\frac{\partial F}{\partial z_{1}} \frac{\partial}{\partial z_{3}}, \frac{\partial}{\partial z_{2}}-\frac{\partial F}{\partial z_{2}} \frac{\partial}{\partial z_{3}}, \frac{\partial}{\partial z_{3}}\right), \\
\tilde{\mathbf{n}}=\frac{\left(-\tilde{\nabla}_{z^{\prime}} F, 1\right)}{\sqrt{\left|\tilde{\nabla}_{z^{\prime}} F\right|^{2}+1}}, \\
\tilde{\Pi} \mathbf{f}=\mathbf{f}-(\tilde{\mathbf{n}} \cdot \mathbf{f}) \tilde{\mathbf{n}}, \\
\tilde{\mathbf{D}}(\boldsymbol{u})=\frac{1}{2}\left(D_{i}^{\prime} u_{j}+D_{j}^{\prime} u_{i}\right)_{i j} .
\end{array}\right.
$$

For the right-hand sides of 440 , we have the following estimate:

$$
\begin{aligned}
&\left\|\mathbf{f}^{\prime}\right\|_{q, \mathbb{R}_{+}^{3}}+\left\|g^{\prime}\right\|_{q, \mathbb{R}_{+}^{3}}^{(1)}+\left\|h^{\prime}\right\|_{q, \mathbb{R}_{+}^{3}}+\left\|\mathbf{F}^{\prime}\right\|_{q, \mathbb{R}^{2}}^{(1-1 / q)}+\left\|F_{3}^{\prime}\right\|_{q, \mathbb{R}^{2}}^{(1-1 / q)}+\left\|G^{\prime}\right\|_{q, \mathbb{R}^{2}}^{(1-1 / q)} \\
& \leqslant C\left\{\|\mathbf{f}\|_{q, B_{\delta}}+\|g\|_{q, B_{\delta}}^{(1)}+\|h\|_{q, B_{\delta}}+\|\mathbf{F}\|_{q, \tilde{B}_{\delta}}^{(1-1 / q)}+\left\|F_{3}\right\|_{q, \tilde{B}_{\delta}}^{(1-1 / q)}+\|G\|_{q, \tilde{B}_{\delta}}^{(1-1 / q)}\right. \\
&\left.+\delta\left([\boldsymbol{u}]_{q, B_{\delta}}^{(2)}+[U]_{q, B_{\delta}}^{(2)}+[q]_{q, B_{\delta}}^{(1)}\right)+\delta^{-2}\left(\|\boldsymbol{u}\|_{q, B_{\delta}}+\|U\|_{q, B_{\delta}}\right)+\delta^{-1}\|q\|_{q, B_{\delta}}\right\},
\end{aligned}
$$

where $B_{\delta}=\left\{z \in \mathbb{R}^{3}|| z \mid \leqslant \delta\right\}$ and $\tilde{B}_{\delta}=\left\{z \in \mathbb{R}^{3}|| z \mid \leqslant \delta, z_{3}=0\right\}$. From the expression $g^{\prime}-\tilde{g}=\nabla \cdot \mathbf{R}$, where

$$
\mathbf{R}=\left(R_{i}\right)=\left(\delta_{i 3} \sum_{j=1,2} F_{z_{j}} \tilde{u}_{j}-\int_{\mathbb{R}_{+}^{3}} \frac{\partial E(z-y)}{\partial z_{i}} \bar{u}(y) \cdot \tilde{\nabla} \phi_{\delta}(y) \mathrm{d} y\right), \quad E(z)=-\frac{1}{4 \pi|z|},
$$

we have the following estimate:

$$
\left\|g^{\prime}\right\|_{q, \mathbb{R}_{+}^{3}}^{(-1)} \leqslant C\left(\delta^{-1}\|\boldsymbol{u}\|_{q, B_{\delta}}^{(-1)}+\|g\|_{q, B_{\delta}}^{(-1)}\right) .
$$


Therefore, applying Theorem 3.5, we have

$$
\begin{aligned}
{[\tilde{\boldsymbol{u}}]_{q, \mathbb{R}_{+}^{3}}^{(2)}+} & |\lambda|\|\tilde{\boldsymbol{u}}\|_{q, \mathbb{R}_{+}^{3}}+\|\nabla \tilde{q}\|_{q, \mathbb{R}_{+}^{3}}+[\tilde{U}]_{q, \mathbb{R}_{+}^{3}}^{(2)}+|\lambda|\|\tilde{U}\|_{q, \mathbb{R}_{+}^{3}} \\
\leqslant & C\left(\|\mathbf{f}\|_{q, B_{\delta}}+[g]_{q, B_{\delta}}^{(1)}+|\lambda|\|g\|_{q, B_{\delta}}^{(-1)}+\|h\|_{q, B_{\delta}}+[\mathbf{F}]_{q, \tilde{B}_{\delta}}^{(1-1 / q)}+|\lambda|^{1 / 2-1 / 2 q}\|\mathbf{F}\|_{q, \tilde{B}_{\delta}}\right. \\
& +\left[F_{3}\right]_{q, \tilde{B}_{\delta}}^{(1-1 / q)}+|\lambda|^{1 / 2-1 / 2 q}\left\|F_{3}\right\|_{q, \tilde{B}_{\delta}}+[G]_{q, \tilde{B}_{\delta}}^{(1-1 / q)}+|\lambda|^{1 / 2-1 / 2 q}\|G\|_{q, \tilde{B}_{\delta}} \\
& \left.+[\boldsymbol{u}]_{q, B_{\delta}}^{(1)}+|\lambda|\|\boldsymbol{u}\|_{q, B_{\delta}}^{(-1)}+\|q\|_{q, B_{\delta}}+[U]_{q, B_{\delta}}^{(1)}+\left(1+|\lambda|^{1 / 2}\right)\|U\|_{q, B_{\delta}}\right) .
\end{aligned}
$$

Similar inequalities can be obtained in the same manner for the solution to the problem localized in $B_{\delta}(x)$, where $x$ is an arbitrary interior point of $\Omega$, which is more distant than $\delta$ from the boundary, or an arbitrary point on $\Sigma$. Since $\Omega$ is bounded, we can choose a finite collection $\left\{B_{\delta}\left(x_{k}\right)\right\}_{k}$ covering $\bar{\Omega}$. Summing all of the estimates over $\left\{B_{\delta}\left(x_{k}\right)\right\}_{k}$, we have

$$
\begin{aligned}
{[\boldsymbol{u}]_{q, \Omega}^{(2)}+} & |\lambda|\|\boldsymbol{u}\|_{q, \Omega}+\|\nabla q\|_{q, \Omega}+[U]_{q, \Omega}^{(2)}+|\lambda|\|U\|_{q, \Omega} \\
\leqslant & C\left(\|\mathbf{f}\|_{q, \Omega}+[g]_{q, \Omega}^{(1)}+|\lambda|\|g\|_{q, \Omega}^{(-1)}+\|h\|_{q, \Omega}+[\mathbf{F}]_{q, \Gamma}^{(1-1 / q)}+|\lambda|^{1 / 2-1 / 2 q}\|\mathbf{F}\|_{q, \Gamma}\right. \\
& +\left[F_{3}\right]_{q, \Gamma}^{(1-1 / q)}+|\lambda|^{1 / 2-1 / 2 q}\left\|F_{3}\right\|_{q, \Gamma}+[G]_{q, \Gamma}^{(1-1 / q)}+|\lambda|^{1 / 2-1 / 2 q}\|G\|_{q, \Gamma}+[H]_{q, \Sigma}^{(2-1 / q)} \\
& \left.+|\lambda|^{1-1 / 2 q}\|H\|_{q, \Sigma}+[\boldsymbol{u}]_{q, \Omega}^{(1)}+|\lambda|\|\boldsymbol{u}\|_{q, \Omega}^{(-1)}+\|q\|_{q, \Omega}+[U]_{q, \Omega}^{(1)}+\left(1+|\lambda|^{1 / 2}\right)\|U\|_{q, \Omega}\right) .
\end{aligned}
$$

Since the solution of problem 90 is unique, we can eliminate the term $[\boldsymbol{u}]_{q, \Omega}^{(1)}+|\lambda|\|\boldsymbol{u}\|_{q, \Omega}^{(-1)}+$ $\|q\|_{q, \Omega}+[U]_{q, \Omega}^{(1)}+\left(1+|\lambda|^{1 / 2}\right)\|U\|_{q, \Omega}$ from the right-hand side. Thus, we have proven Theorem 3.1.

To end this section, we state a result concerning the following nonstationary problem:

$$
\left\{\begin{array}{l}
\frac{\partial \boldsymbol{u}}{\partial t}-v \Delta \boldsymbol{u}+c_{0} \nabla q=\mathbf{f}, \quad \nabla \cdot \boldsymbol{u}=0 \\
\frac{\partial U}{\partial t}-\chi \Delta U=h \quad \text { in } \Omega_{\infty} \\
2 \nu \Pi \mathbf{D}(\boldsymbol{u}) \mathbf{n}=\mathbf{0}, \quad 2 \nu \mathbf{D}(\boldsymbol{u}) \mathbf{n} \cdot \mathbf{n}-c_{0} q-c_{1} U=0 \\
\nabla U \cdot \mathbf{n}+c_{2} \boldsymbol{u} \cdot \mathbf{n}=0 \quad \text { on } \Gamma_{\infty} \\
\boldsymbol{u}=\mathbf{0}, \quad U=0 \quad \text { on } \Sigma_{\infty} \\
\left.(\boldsymbol{u}, U)\right|_{t=0}=\left(\boldsymbol{u}_{0}, U_{0}\right) \quad \text { on } \Omega
\end{array}\right.
$$

Let us formulate the problem as an evolution equation. Let us introduce a decomposition $L_{q}(\Omega)=$ $J_{q}(\Omega) \oplus G_{q}(\Omega)$, where $J_{q}(\Omega)=\left\{\boldsymbol{u} \in L_{q}(\Omega)|\nabla \cdot \boldsymbol{u}=0, \boldsymbol{u} \cdot \mathbf{n}|_{\Sigma}=0\right\}$ and $G_{q}(\Omega)=\{\nabla p \mid$ $\left.p \in W_{q}^{1}(\Omega),\left.p\right|_{\Gamma}=0\right\}$, and let us introduce the projection operator $P$ on $J_{q}(\Omega)$. Then, applying $P$ to problem 41 , we have the evolution equation

$$
\left\{\begin{array}{l}
(\boldsymbol{u}, U)_{t}=A(\boldsymbol{u}, U)+(\mathbf{f}, h) \quad \text { in } t>0, \\
\left.(\boldsymbol{u}, U)\right|_{t=0}=\left(\boldsymbol{u}_{0}, U_{0}\right)
\end{array}\right.
$$

where $A(\boldsymbol{u}, U)$ is the operator $(-P(v \Delta \boldsymbol{u})+\nabla \tilde{q}, \chi \Delta U)$ defined on 


$$
\begin{array}{r}
\mathcal{D}(A)=\left\{(\boldsymbol{u}, U) \in\left(J_{q}(\Omega) \cap W_{q}^{2}(\Omega)\right) \times W_{q}^{2}(\Omega)\left|2 v \mathbf{D}(\boldsymbol{u}) \mathbf{n}-c_{0} \tilde{q} \mathbf{n}-c_{1} U \mathbf{n}\right|_{\Gamma}=\mathbf{0},\right. \\
\left.\nabla U \cdot \mathbf{n}+\left.c_{2} \boldsymbol{u} \cdot \mathbf{n}\right|_{\Gamma}=0,\left.\boldsymbol{u} \cdot \mathbf{n}\right|_{\Sigma}=0,\left.\quad U\right|_{\Sigma}=0\right\},
\end{array}
$$

and $\tilde{q}$ is the solution of the problem

$$
\left\{\begin{array}{l}
\Delta \tilde{q}=0 \quad \text { in } \Omega, \\
c_{0} \tilde{q}=2 v \mathbf{D}(\boldsymbol{u}) \mathbf{n} \cdot \mathbf{n}-\left.c_{1} U\right|_{\Gamma} \quad \text { on } \Gamma, \\
\nabla \tilde{q} \cdot \mathbf{n}=0 \quad \text { on } \Sigma .
\end{array}\right.
$$

From Theorems 3.1 and 3.2, we can obtain the following theorem.

Theorem 3.6 Let $1<q<\infty$. Then $A$ generates an analytic semigroup $\left\{e^{-A t}\right\}_{t \geqslant 0}$ with the property of exponential stability.

\section{Linear problem}

In this section, we consider the following nonstationary linear problem:

$$
\left\{\begin{array}{l}
\frac{\partial \boldsymbol{v}}{\partial t}-v \Delta \boldsymbol{v}+c_{0} \nabla p=\mathbf{f}, \quad \nabla \cdot \boldsymbol{v}=g \\
\frac{\partial T}{\partial t}-\chi \Delta T=h \quad \text { in } \Omega_{\infty}, \\
2 v \Pi \mathbf{D}(\boldsymbol{v}) \mathbf{n}=\mathbf{F}\left(=\left(F_{1}, F_{2}\right)\right), \quad 2 \nu \mathbf{D}(\boldsymbol{v}) \mathbf{n} \cdot \mathbf{n}-c_{0} p-c_{1} T=F_{3} \\
\nabla T \cdot \mathbf{n}+c_{2} \boldsymbol{v} \cdot \mathbf{n}=G \quad \text { on } \Gamma_{\infty}, \\
\boldsymbol{v}=\mathbf{0}, \quad T=H \quad \text { on } \Sigma_{\infty}, \\
\left.\boldsymbol{v}\right|_{t=0}=\boldsymbol{v}_{0},\left.\quad T\right|_{t=0}=T_{0} \quad \text { on } \Omega .
\end{array}\right.
$$

The goal is to prove the following maximal regularity result.

Theorem 4.1 Let $1<q<\infty$. Assume that

$$
\begin{gathered}
\mathbf{f} \in L_{q}\left(\Omega_{\infty}\right), \quad g \in W_{q}^{1,0}\left(\Omega_{\infty}\right), \quad h \in L_{q}\left(\Omega_{\infty}\right), \quad H \in W_{q}^{2-1 / q, 1-1 / 2 q}\left(\Sigma_{\infty}\right), \\
\mathbf{F}=\left(F_{1}, F_{2}\right), F_{3} \in W_{q}^{1-1 / q, 1 / 2-1 / 2 q}\left(\Gamma_{\infty}\right), \quad G \in W_{q}^{1-1 / q, 1 / 2-1 / 2 q}\left(\Gamma_{\infty}\right), \\
\boldsymbol{v}_{0} \in W_{q}^{2-2 / q}(\Omega), \quad T_{0} \in W_{q}^{2-2 / q}(\Omega),
\end{gathered}
$$

and $g=\nabla \cdot \mathbf{R}, \mathbf{R} \in W_{q}^{1}\left(0, \infty ; L_{q}(\Omega)\right)$. Then problem 42 has a unique solution

$$
(\boldsymbol{v}, p, T) \in W_{q}^{2,1}\left(\Omega_{\infty}\right) \times W_{q}^{1,0}\left(\Omega_{\infty}\right) \times W_{q}^{2,1}\left(\Omega_{\infty}\right)
$$

satisfying the estimate

$$
\begin{aligned}
\|\boldsymbol{v}\|_{q, \Omega_{\infty}}^{(2,1)} & +\|p\|_{q, \Omega_{\infty}}^{(1,0)}+\|T\|_{q, \Omega_{\infty}}^{(2,1)} \\
\leqslant & C\left(\|\mathbf{f}\|_{q, \Omega_{\infty}}+\|g\|_{q, \Omega_{\infty}}^{(1,0)}+\|\mathbf{R}\|_{q, \Omega_{\infty}}^{(0,1)}+\|h\|_{q, \Omega_{\infty}}+\left\|\boldsymbol{v}_{0}\right\|_{q, \Omega}^{(2-2 / q)}+\left\|T_{0}\right\|_{q, \Omega}^{(2-2 / q)}\right. \\
& +\|\mathbf{F}\|_{q, \Gamma_{\infty}}^{(1-1 / q, 1 / 2-1 / 2 q)}+\left\|F_{3}\right\|_{q, \Gamma_{\infty}}^{(1-1 / q, 1 / 2-1 / 2 q)}+\|G\|_{q, \Gamma_{\infty}}^{(1-1 / q, 1 / 2-1 / 2 q)} \\
& \left.+\|H\|_{q, \Sigma_{\infty}}^{(2-1 / q, 1-1 / 2 q)}\right),
\end{aligned}
$$

where $C=C(q, \Omega)$ is a positive constant. 
We begin by investigating the following model problems:

$$
\begin{aligned}
& \left\{\begin{array}{l}
\frac{\partial \boldsymbol{u}}{\partial t}-v \Delta \boldsymbol{u}+c_{0} \nabla q=\mathbf{f}, \quad \nabla \cdot \boldsymbol{u}=g, \\
\frac{\partial U}{\partial t}-\chi \Delta U=h \quad \text { in } \mathbb{R}_{\infty}^{3} \equiv \mathbb{R}^{3} \times(0, \infty), \\
\left.\boldsymbol{u}\right|_{t=0}=\mathbf{0},\left.\quad T\right|_{t=0}=0 \quad \text { on } \mathbb{R}^{3},
\end{array}\right. \\
& \left\{\begin{array}{l}
\frac{\partial \boldsymbol{u}}{\partial t}-v \Delta \boldsymbol{u}+c_{0} \nabla q=\mathbf{f}, \quad \nabla \cdot \boldsymbol{u}=g, \\
\frac{\partial U}{\partial t}-\chi \Delta U=h \quad \text { in } \mathbb{D}_{\infty}^{3} \equiv \mathbb{R}_{+}^{3} \times(0, \infty), \\
\left.\boldsymbol{u}\right|_{z_{3}=0}=\mathbf{I},\left.\quad U\right|_{z_{3}=0}=H \quad \text { on } \mathbb{R}_{\infty}^{2}, \\
\left.\boldsymbol{u}\right|_{t=0}=\mathbf{0},\left.\quad U\right|_{t=0}=0 \quad \text { on } \mathbb{R}_{+}^{3},
\end{array}\right.
\end{aligned}
$$

and

$$
\left\{\begin{array}{l}
\frac{\partial \boldsymbol{u}}{\partial t}-v \Delta \boldsymbol{u}+c_{0} \nabla q=\mathbf{f}, \quad \nabla \cdot \boldsymbol{u}=g \\
\frac{\partial U}{\partial t}-\chi \Delta U=h \quad \text { in } \mathbb{D}_{\infty}^{3}, \\
\left.v\left(\frac{\partial u_{i}}{\partial z_{3}}+\frac{\partial u_{3}}{\partial z_{i}}\right)\right|_{z_{3}=0}=F_{i}(i=1,2), \quad 2 v \frac{\partial u_{3}}{\partial z_{3}}-c_{0} q-\left.c_{1} U\right|_{z_{3}=0}=F_{3}, \\
\frac{\partial U}{\partial z_{3}}+\left.c_{2} u_{3}\right|_{z_{3}=0}=G \quad \text { on } \mathbb{R}_{\infty}^{2}, \\
\left.\boldsymbol{u}\right|_{t=0}=\mathbf{0},\left.\quad U\right|_{t=0}=0 \quad \text { on } \mathbb{R}_{+}^{3} .
\end{array}\right.
$$

For the above problems, we have the following theorems. Theorems 4.2 and 4.3 can be proven in essentially the same manner as Theorem 4.4, and these proofs are simpler than the proof of Theorem 4.4. Therefore, we present only the proof of Theorem 4.4.

THEOREM 4.2 Let $1<q<\infty$. Suppose that

$$
\mathbf{f} \in L_{q}\left(\mathbb{R}_{\infty}^{3}\right), \quad g \in L_{q}\left(0, \infty ; W_{q}^{1}\left(\mathbb{R}^{3}\right)\right) \cap W_{q}^{1}\left(0, \infty ; \dot{W}_{q}^{-1}\left(\mathbb{R}^{3}\right)\right), \quad h \in L_{q}\left(\mathbb{R}_{\infty}^{3}\right),
$$

and that $g$ has a compact support. In addition, assume that the solution $(\boldsymbol{u}, q, U)$ of problem (44) tends toward 0 as $|z| \rightarrow \infty$. Then problem (44) has a unique solution satisfying the following estimate:

$$
[\boldsymbol{u}]_{q, \mathbb{R}_{\infty}^{3}}^{(2,1)}+\|\nabla q\|_{q, \mathbb{R}_{\infty}^{3}}+[U]_{q, \mathbb{R}_{\infty}^{3}}^{(2,1)} \leqslant C\left(\|\mathbf{f}\|_{q, \mathbb{R}_{\infty}^{3}}+\left\|g_{t}\right\|_{q, \mathbb{R}_{\infty}^{3}}^{(0,-1)}+[g]_{q, \mathbb{R}_{\infty}^{3}}^{(1,0)}+\|h\|_{q, \mathbb{R}_{\infty}^{3}}\right)
$$

where $C=C(q)$ is a positive constant.

THEOREM 4.3 Let $1<q<\infty$. Suppose that

$$
\begin{gathered}
\mathbf{f} \in L_{q}\left(\mathbb{D}_{\infty}^{3}\right), \quad g \in L_{q}\left(0, \infty ; W_{q}^{1}\left(\mathbb{R}_{+}^{3}\right)\right) \cap W_{q}^{1}\left(0, \infty ; \dot{W}_{q}^{-1}\left(\mathbb{R}_{+}^{3}\right)\right), \\
h \in L_{q}\left(\mathbb{D}_{\infty}^{3}\right), \quad \mathbf{I} \in W_{q}^{2-1 / q, 1-1 / 2 q}\left(\mathbb{R}_{\infty}^{2}\right), \quad H \in W_{q}^{2-1 / q, 1-1 / 2 q}\left(\mathbb{R}_{\infty}^{2}\right),
\end{gathered}
$$


and that $g$ has a compact support. In addition, assume that the solution $(\boldsymbol{u}, q, U)$ of problem (45) tends toward 0 as $z_{3} \rightarrow \infty$. Then problem (45) has a unique solution satisfying the following estimate:

$$
\begin{aligned}
{[\boldsymbol{u}]_{q, \mathbb{D}_{\infty}^{3}}^{(2,1)}+\|\nabla q\|_{q, \mathbb{D}_{\infty}^{3}}+[U]_{q, \mathbb{D}_{\infty}^{3}}^{(2,1)} \leqslant } & C\left(\|\mathbf{f}\|_{q, \mathbb{D}_{\infty}^{3}}+\left\|g_{t}\right\|_{q, \mathbb{D}_{\infty}^{3}}^{(0,-1)}+[g]_{q, \mathbb{D}_{\infty}^{3}}^{(1,0)}+\|h\|_{q, \mathbb{D}_{\infty}^{3}}\right. \\
& \left.+[\mathbf{I}]_{q, \mathbb{R}_{\infty}^{2}}^{(2-1 / q, 1-1 / 2 q)}+[H]_{q, \mathbb{R}_{\infty}^{2}}^{(2-1 / q, 1-1 / 2 q)}\right),
\end{aligned}
$$

where $C=C(q)$ is a positive constant.

THEOREM 4.4 Let $1<q<\infty$. Suppose that

$$
\begin{gathered}
\mathbf{f} \in L_{q}\left(\mathbb{D}_{\infty}^{3}\right), \quad g \in L_{q}\left(0, \infty ; W_{q}^{1}\left(\mathbb{R}_{+}^{3}\right)\right) \cap W_{q}^{1}\left(0, \infty ; \dot{W}_{0, q}^{-1}\left(\mathbb{R}_{+}^{3}\right)\right), \quad h \in L_{q}\left(\mathbb{D}_{\infty}^{3}\right), \\
F_{1}, F_{2}, F_{3} \in W_{q}^{1-1 / q, 1 / 2-1 / 2 q}\left(\mathbb{R}_{\infty}^{2}\right), \quad G \in W_{q}^{1-1 / q, 1 / 2-1 / 2 q}\left(\mathbb{R}_{\infty}^{2}\right),
\end{gathered}
$$

and that $g$ has a compact support. In addition, assume that the solution $(\boldsymbol{u}, q, U)$ of problem (46) tends toward 0 as $z_{3} \rightarrow \infty$. Then problem (46) has a unique solution satisfying the following estimate:

$$
\begin{aligned}
{[\boldsymbol{u}]_{q, \mathbb{D}_{\infty}^{3}}^{(2,1)}+\|\nabla q\|_{q, \mathbb{D}_{\infty}^{3}}+[U]_{q, \mathbb{D}_{\infty}^{3}}^{(2,1)} \leqslant } & C\left(\|\mathbf{f}\|_{q, \mathbb{D}_{\infty}^{3}}+\left\|g_{t}\right\|_{q, \mathbb{D}_{\infty}^{3}}^{(0,-1)}+[g]_{q, \mathbb{D}_{\infty}^{3}}^{(1,0)}+\|h\|_{q, \mathbb{D}_{\infty}^{3}}\right. \\
& \left.+\sum_{i=1}^{3}\left[F_{i}\right]_{q, \mathbb{R}_{\infty}^{2}}^{(1-1 / q, 1 / 2-1 / q)}+[G]_{q, \mathbb{R}_{\infty}^{2}}^{(1-1 / 2,1 / 2-1 / q)}\right),
\end{aligned}
$$

where $C=C(q)$ is a positive constant.

Proof. In the proof, we denote various constants that are independent of $t$ by $C$. Let us seek the solution in the form

$$
(\boldsymbol{u}, q, U)=\left(\mathbf{W}+\nabla \Phi+\boldsymbol{w}, \pi-\frac{1}{c_{0}}\left(\frac{\partial \Phi}{\partial t}-\nu \Delta \Phi\right), \mathcal{U}+\theta\right),
$$

where $\mathbf{W}, \Phi, \mathcal{U}$ and $(\boldsymbol{w}, \pi, \theta)$ are solutions to the following problems:

$$
\begin{aligned}
& \left\{\begin{array}{l}
\frac{\partial \mathbf{W}}{\partial t}-v \Delta \mathbf{W}=\mathbf{f} \quad \text { in } \mathbb{D}_{\infty}^{3} \\
\left.\mathbf{W}\right|_{z_{3}=0}=\mathbf{0} \quad \text { on } \mathbb{R}_{\infty}^{2} \\
\left.\mathbf{W}\right|_{t=0}=\mathbf{0} \quad \text { on } \mathbb{R}_{+}^{3}
\end{array}\right. \\
& \left\{\begin{array}{l}
\Delta \Phi=g-\nabla \cdot \mathbf{W} \equiv g^{\prime} \quad \text { in } \mathbb{D}_{\infty}^{3}, \\
\left.\Phi\right|_{z_{3}=0}=0 \quad \text { on } \mathbb{R}_{\infty}^{2},
\end{array}\right. \\
& \left\{\begin{array}{l}
\frac{\partial \mathcal{U}}{\partial t}-\chi \Delta \mathcal{U}=h \quad \text { in } \mathbb{D}_{\infty}^{3}, \\
\left.\frac{\partial \mathcal{U}}{\partial z_{3}}\right|_{z_{3}=0}=G-\left.c_{2}\left(\frac{\partial \Phi}{\partial z_{3}}+W_{3}\right)\right|_{z_{3}=0} \equiv G^{\prime} \quad \text { on } \mathbb{R}_{\infty}^{2}, \\
\left.\mathcal{U}\right|_{t=0}=0 \text { on } \mathbb{R}_{+}^{3},
\end{array}\right.
\end{aligned}
$$




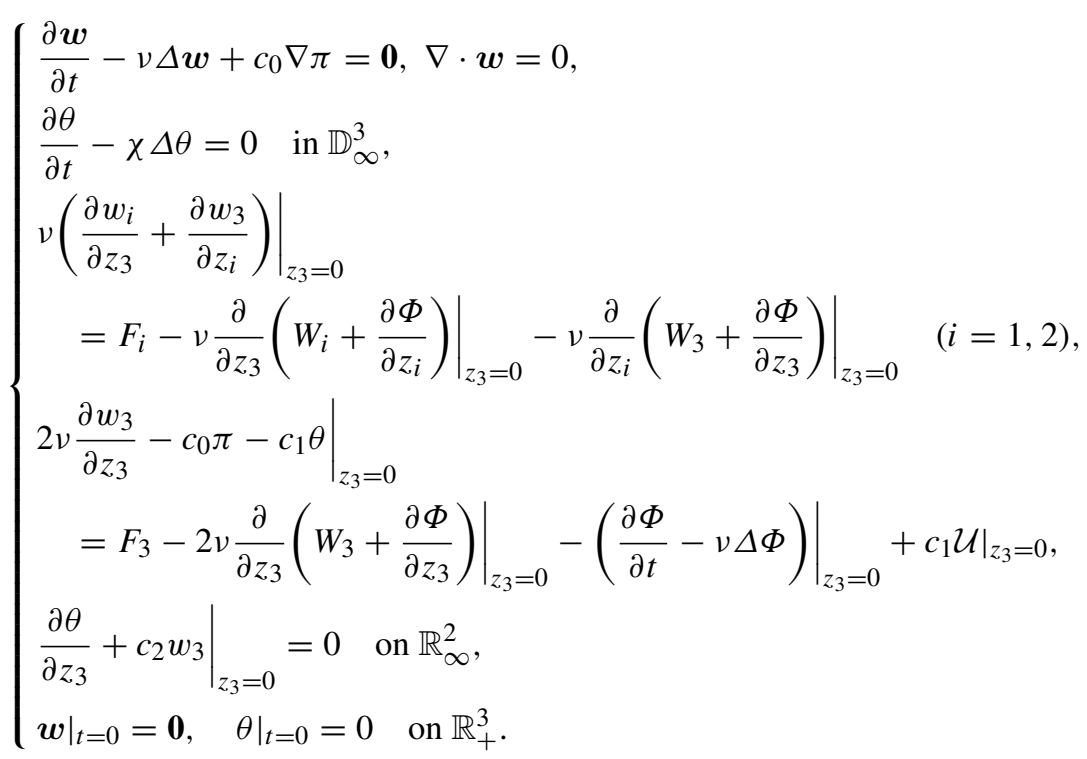

For $\mathbf{W}, \Phi, \mathcal{U}$, the following estimates are easily obtained:

$$
\begin{aligned}
{[\mathbf{W}]_{q, \mathbb{D}_{\infty}^{3}}^{(2,1)} } & \leqslant C\|\mathbf{f}\|_{q, \mathbb{D}_{\infty}^{3}}, \\
{[\nabla \Phi]_{q, \mathbb{D}_{\infty}^{3}}^{(2,1)} } & \leqslant C\left(\left[g^{\prime}\right]_{q, \mathbb{D}_{\infty}^{3}}^{(1,0)}+\left\|g_{t}^{\prime}\right\|_{q, \mathbb{D}_{\infty}^{3}}^{(0,-1)}\right), \\
{[\mathcal{U}]_{q, \mathbb{D}_{\infty}^{3}}^{(2,1)} } & \leqslant C\left(\|h\|_{q, \mathbb{D}_{\infty}^{3}}+\left[G^{\prime}\right]_{\mathbb{R}_{\infty}^{2}}^{(1-1 / q, 1 / 2-1 / 2 q)}\right) .
\end{aligned}
$$

Let us next estimate the solution of problem (53). We denote the terms on the right-hand side by $F_{i}, i=1,2,3$. The solution is given as follows:

$$
\begin{aligned}
w_{i} & =(\mathcal{F} \mathcal{L})^{-1}\left(\tilde{W}_{i}\right)+\mathcal{L}^{-1}\left(\mathcal{W}_{i}\right), \\
q & =(\mathcal{F} \mathcal{L})^{-1}(\tilde{P})+\mathcal{L}^{-1}(\mathcal{P}), \\
\theta & =(\mathcal{F} \mathcal{L})^{-1}\left(\sum_{j=1}^{3} \tilde{G}_{j} e^{-R z_{3}} \tilde{F}_{j}\right),
\end{aligned}
$$

where $\tilde{W}_{i}$ and $\tilde{P}$ are given in 31 and $\mathcal{W}_{i}$ and $\mathcal{P}$ are given in 37 . In the above formula, $\mathcal{L}^{-1}$ and $(\mathcal{F} \mathcal{L})^{-1}$ denote the inverse Laplace transformation and the inverse Fourier-Laplace transformation, respectively, which are defined as follows:

$$
\begin{aligned}
\mathcal{L}^{-1}(f)(z, t) & \equiv \frac{1}{2 \pi i} \int_{a-i \infty}^{a+i \infty} e^{\lambda t} f(z, \lambda) \mathrm{d} \lambda \\
(\mathcal{F} \mathcal{L})^{-1}(f)(z, t) & \equiv \frac{1}{(2 \pi) 2 \pi i} \int_{\mathbb{R}^{2}} e^{i z^{\prime} \cdot \xi^{\prime}} \mathrm{d} \xi^{\prime} \int_{a-i \infty}^{a+i \infty} e^{\lambda t} f\left(\xi^{\prime}, z_{3}, \lambda\right) \mathrm{d} \lambda .
\end{aligned}
$$

For $(\mathcal{F} \mathcal{L})^{-1}\left(\tilde{W}_{i}\right)$ and $(\mathcal{F} \mathcal{L})^{-1}(\tilde{P})$, the estimate

$$
\sum_{i=1}^{3}\left[(\mathcal{F} \mathcal{L})^{-1}\left(\tilde{W}_{i}\right)\right]_{q, \mathbb{D}_{\infty}^{3}}^{(2,1)}+\left\|\nabla(\mathcal{F} \mathcal{L})^{-1}(\tilde{P})\right\|_{q, \mathbb{D}_{\infty}^{3}} \leqslant C \sum_{i=1}^{3}\left[F_{i}\right]_{q, \mathbb{R}_{\infty}^{2}}^{(1-1 / q, 1 / 2-1 / 2 q)}
$$

has been reported in [22]. 
Let us next estimate the remaining terms. We begin with the derivation of the following estimate of the kernel $\mathcal{G}_{i}(z, t)=(\mathcal{F} \mathcal{L})^{-1}\left(\tilde{G}_{i}\right)$, where $\tilde{G}_{i}$ is given in 30 .

LEMMA 4.1 The following inequalities hold:

$$
\left|D_{t}^{\mu} D_{z}^{v} \mathcal{G}_{i}(z, t)\right| \leqslant C t^{-\mu-|\nu| / 2-3 / 2} e^{-c|z|^{2} / t} \quad(i=1,2,3) .
$$

First, we prove the following lemma that is essential to obtaining estimate (58).

LEMma 4.2 The roots $\lambda$ of $R Q+2 c_{1} c_{2}\left|\xi^{\prime}\right|\left(r+\left|\xi^{\prime}\right|\right)$ satisfy the following condition:

$$
\operatorname{Re} \lambda \leqslant-\delta\left|\xi^{\prime}\right|^{2}
$$

for a positive constant $\delta$.

Proof. Set $l_{\delta} \equiv\left\{\left.\lambda|\operatorname{Re} \lambda=-\delta| \xi^{\prime}\right|^{2}\right\}$ for a positive constant $\delta$. On $l_{\delta}, r$ is written as $r=\sqrt{(1-\delta / v)\left|\xi^{\prime}\right|^{2}+i \operatorname{Im} \lambda / v}$. Then, for arbitrary $\delta<v$, we have $|\arg r|<\pi / 4$, and $R$ has the same property: for arbitrary $\delta<\chi$, we have $|\arg R|<\pi / 4$ on $l_{\delta}$. These properties imply $\left|\arg (R Q)-\arg \left(2 c_{1} c_{2}\left|\xi^{\prime}\right|\left(r+\left|\xi^{\prime}\right|\right)\right)\right|<\pi$, and so

$$
\left|R Q+2 c_{1} c_{2}\right| \xi^{\prime}\left|\left(r+\left|\xi^{\prime}\right|\right)\right| \geqslant C\left(|R Q|+\left|\xi^{\prime}\right||r+| \xi^{\prime} \mid\right)
$$

on $l_{\delta}$ for some positive constant $C$. Since the roots of $R Q$ and $\left|\xi^{\prime}\right|\left(r+\left|\xi^{\prime}\right|\right)$ satisfy $\operatorname{Re} \lambda \leqslant-\delta^{\prime}\left|\xi^{\prime}\right|^{2}$ for a positive constant $\delta^{\prime}$, the inequality 600 shows that $\left|R Q+2 c_{1} c_{2}\right| \xi^{\prime}\left|\left(r+\left|\xi^{\prime}\right|\right)\right|$ has no roots on $l_{\delta}$ for arbitrary $\delta \leqslant \min \left(\nu, \chi, \delta^{\prime}\right)$. This implies that the lemma holds.

Now, let us proceed to the proof of Lemma 4.1. We shall give the proof only for $\mathcal{G}_{3}$. The estimates for $\mathcal{G}_{1}$ and $\mathcal{G}_{2}$ are derived in the same manner. First, we set

$$
\lambda t=\lambda^{\prime}, \quad \xi^{\prime} \sqrt{t}=\eta^{\prime}, \quad z^{\prime}=\sqrt{t} \zeta^{\prime}, \quad z_{3}=\sqrt{t} \zeta_{3} .
$$

Then we have

$$
\mathcal{G}_{3}=t^{-\mu-|\nu| / 2-3 / 2} G_{\mu, \nu},
$$

where

$$
G_{\mu, v}=\int_{a+i \infty}^{a+i \infty} \lambda^{\prime \mu} e^{\lambda^{\prime}} \mathrm{d} \lambda^{\prime} \int_{\mathbb{R}^{2}} e^{i \zeta^{\prime} \cdot \eta^{\prime}} \frac{\left(i \eta^{\prime}\right)^{v^{\prime}} c_{1} c_{2}\left|\eta^{\prime}\right|\left(r+\left|\eta^{\prime}\right|\right)(-R)^{\nu_{3}}}{v\left(t^{-1 / 2} R Q+2 c_{1} c_{2} t^{1 / 2}\left|\eta^{\prime}\right|\left(r+\left|\eta^{\prime}\right|\right)\right)} e^{-R \zeta_{3}} \mathrm{~d} \eta^{\prime}
$$

Lemma 4.2 indicates that it is possible to replace the path of integration $\left\{\lambda^{\prime} \mid \operatorname{Re} \lambda^{\prime}=a\right\}$ by $l(a) \equiv\left\{\left.\lambda^{\prime}\left|\operatorname{Re} \lambda^{\prime}=a-\delta\right| \eta^{\prime}\right|^{2}-\epsilon\left|\operatorname{Im} \lambda^{\prime}\right|\right\}$ for sufficiently small $\delta$ and $\epsilon$. Therefore, we estimate the following integral:

$$
G_{\mu, v}=\int_{l(a)} \lambda^{\prime \mu} e^{\lambda^{\prime}} \mathrm{d} \lambda^{\prime} \int_{\mathbb{R}^{2}} e^{i \zeta^{\prime} \cdot \eta^{\prime}} \frac{\left(i \eta^{\prime}\right)^{\nu^{\prime}} c_{1} c_{2}\left|\eta^{\prime}\right|\left(r+\left|\eta^{\prime}\right|\right)(-R)^{\nu_{3}}}{v\left(t^{-1 / 2} R Q+2 c_{1} c_{2} t^{1 / 2}\left|\eta^{\prime}\right|\left(r+\left|\eta^{\prime}\right|\right)\right)} e^{-R \zeta_{3}} \mathrm{~d} \eta^{\prime}
$$

First, we prove that

$$
\left|G_{\mu, v}\right| \leqslant C e^{-c\left|\zeta^{\prime}\right|^{2}}
$$

Here, we introduce the new variables $\alpha^{\prime} \equiv \eta^{\prime}\left|\zeta^{\prime}\right|^{-1}$ and $p^{\prime}=\lambda^{\prime}\left|\zeta^{\prime}\right|^{-2}$ and shift the path of integration from $l\left(a /\left|\zeta^{\prime}\right|^{2}\right)$ to $l(a)$. Then we have 
$G_{\mu, \nu}$

$$
=\int_{l(a)}\left|\zeta^{\prime}\right|^{2 \mu} p^{\prime \mu} e^{\left|\zeta^{\prime}\right|^{2} p^{\prime}} \mathrm{d} p^{\prime} \int_{\mathbb{R}^{2}} e^{i\left|\zeta^{\prime}\right|\left(\alpha^{\prime} \cdot \zeta^{\prime}\right)} \frac{\left|\zeta^{\prime}\right|^{\left|\nu^{\prime}\right|+3+\nu_{3}}\left(i \alpha^{\prime}\right)^{\nu^{\prime}} c_{1} c_{2}\left|\alpha^{\prime}\right|\left(r+\left|\alpha^{\prime}\right|\right)(-R)^{\nu_{3}}}{\nu\left(t^{-1 / 2}\left|\zeta^{\prime}\right| R Q+2 c_{1} c_{2} t^{1 / 2}\left|\alpha^{\prime}\right|\left(r+\alpha^{\prime} \mid\right)\right)} e^{-R \zeta_{3}} \mathrm{~d} \alpha^{\prime} .
$$

We then shift the integration contour $\alpha^{\prime}$ to $\beta^{\prime}+i \gamma^{\prime}$. By elementary calculations, it is easily verified that if we choose $\gamma^{\prime}=\left(\gamma_{1}^{\prime}, \gamma_{2}^{\prime}\right)$ satisfying $\left|\gamma^{\prime}\right| \leqslant \sqrt{a /(\nu+\delta)}$, then $|\arg r|<\pi / 4$. Thus, from 60 , if we take $\rho \sqrt{a / M} \zeta^{\prime} /\left|\zeta^{\prime}\right|$ as $\gamma^{\prime}$, where $M=\max (v+\delta, \chi+\delta)$ and $\rho$ is a sufficiently small positive constant, then $\left|R Q+c_{1} c_{2}\right| \xi^{\prime}\left|\left(r+\left|\xi^{\prime}\right|\right)\right| \geqslant C|R Q|^{1 / 2}\left|\xi^{\prime}\right|^{1 / 2}|r+| \xi^{\prime}||^{1 / 2}$ on this integration contour. Thus, using the inequalities

$$
\left\{\begin{array}{l}
|Q| \geqslant C\left(1+\left|\beta^{\prime}\right|^{2}+\left|\operatorname{Im} p^{\prime}\right|\right)^{3 / 2} \\
|R| \geqslant C\left(1+\left|\beta^{\prime}\right|^{2}+\left|\operatorname{Im} p^{\prime}\right|\right)^{1 / 2} \\
|r+| \alpha^{\prime}||,|R| \leqslant C\left(1+\left|\beta^{\prime}\right|^{2}+\left|\operatorname{Im} p^{\prime}\right|\right)^{1 / 2}
\end{array}\right.
$$

we have

$$
\begin{aligned}
& \left.\left|\int_{l(a)}\right| \zeta^{\prime}\right|^{2 \mu+2}\left|p^{\prime}\right|^{\mu} e^{\left|\zeta^{\prime}\right|^{2} p^{\prime}} e^{i\left|\zeta^{\prime}\right|\left(\beta^{\prime}+i \gamma^{\prime} \cdot \zeta^{\prime}\right)} \frac{\left|\zeta^{\prime}\right|^{\left|v^{\prime}\right|+v_{3}+4}\left|\beta^{\prime}+i \gamma^{\prime}\right|^{\left|v^{\prime}\right|+1}|| \beta^{\prime}+i \gamma^{\prime}|+r||R|^{\nu_{3}}}{\left|\zeta^{\prime}\right|^{3}|R Q|^{1 / 2}\left|\beta^{\prime}+i \gamma^{\prime}\right|^{1 / 2}|r+| \beta^{\prime}+i \gamma^{\prime}||^{1 / 2}} \mathrm{~d} p^{\prime} \mid \\
& \leqslant C\left|\zeta^{\prime}\right|^{2 \mu+\left|v^{\prime}\right|+\nu_{3}+3} e^{\left(a+\delta \rho^{2} a / M-\rho \sqrt{a / M}\right)}\left|\zeta^{\prime}\right|^{2} \\
& \quad \times\left.\left|\int_{0}^{\infty}\right| \operatorname{Im} p^{\prime}\right|^{\mu} e^{-\chi\left|\operatorname{Im} p^{\prime}\right|} \frac{\left(1+\left|\beta^{\prime}\right|\right)^{2 \mu+\left|v^{\prime}\right|+1 / 2}\left(1+\left|\beta^{\prime}\right|^{2}+\left|\operatorname{Im} p^{\prime}\right|\right)^{1 / 2+v_{3}}}{1+\left|\beta^{\prime}\right|^{2}+\left|\operatorname{Im} p^{\prime}\right|} e^{-\delta\left|\beta^{\prime}\right|^{2}\left|\zeta^{\prime}\right|^{2}} \mathrm{~d}\left|\operatorname{Im} p^{\prime}\right| \\
& \leqslant\left|\zeta^{\prime}\right|^{2 \mu+\left|v^{\prime}\right|+v_{3}+3} e^{\left(a+\delta \rho^{2} a / M-\rho \sqrt{a / M}\right)\left|\zeta^{\prime}\right|^{2}}\left(1+\left|\beta^{\prime}\right|\right)^{2 \mu+\left|v^{\prime}\right|+v_{3}-1} e^{-\delta\left|\beta^{\prime}\right|^{2}\left|\zeta^{\prime}\right|^{2}} .
\end{aligned}
$$

As a consequence of this estimate, we have

$$
\begin{aligned}
\left|G_{\mu, \nu}\right| & \leqslant C\left|\zeta^{\prime}\right|^{2 \mu+\left|v^{\prime}\right|+v_{3}+3} e^{\left(a+\delta \rho^{2} a / M-\rho \sqrt{a / M}\right)\left|\zeta^{\prime}\right|^{2}} \int_{\mathbb{R}^{2}}\left(1+\left|\beta^{\prime}\right|\right)^{2 \mu+\left|v^{\prime}\right|+v_{3}-1} e^{-\delta\left|\beta^{\prime}\right|^{2}\left|\zeta^{\prime}\right|^{2}} \mathrm{~d} \beta^{\prime} \\
& \leqslant C\left|\zeta^{\prime}\right|^{2} e^{\left(a+\delta \rho^{2} a / M-\rho \sqrt{a / M}\right)\left|\zeta^{\prime}\right|^{2}}
\end{aligned}
$$

Since $a+\delta \rho^{2} a / M-\rho \sqrt{a / M}<0$ for sufficiently small $a$, if we choose such an $a$, we have the estimate given in 61,.

Next, we prove that

$$
\left|G_{\mu, \nu}\right| \leqslant C e^{-c \zeta_{3}^{2}}
$$

For this purpose, we introduce the new variables $q=\zeta_{3}^{-2} \lambda^{\prime}$ and $\alpha^{\prime}=\zeta_{3}^{-1} \eta^{\prime}$. After changing the variables and shifting the path of integration from $l\left(a / \zeta_{3}^{2}\right)$ to $l(a)$, we have

$$
G_{\mu, v}=\int_{l(a)} \zeta_{3}^{2 \mu+2} q^{\mu} e^{\zeta_{3}^{2} q} \mathrm{~d} q \int_{\mathbb{R}^{2}} \frac{\zeta_{3}^{\left|v^{\prime}\right|+\nu_{3}+4}\left(i \alpha^{\prime}\right)^{v^{\prime}} c_{1} c_{2}\left|\alpha^{\prime}\right|\left(r+\left|\alpha^{\prime}\right|\right)(-R)^{\nu_{3}}}{v\left(\zeta_{3}^{4} t^{-1 / 2} R Q+2 c_{1} c_{2} \zeta_{3}^{2} t^{1 / 2}\left|\alpha^{\prime}\right|\left(r+\left|\alpha^{\prime}\right|\right)\right)} e^{-R \zeta_{3}^{2}} \mathrm{~d} \alpha^{\prime} .
$$

Using the estimate $\left|R Q+2 c_{1} c_{2}\right| \xi^{\prime}\left|\left(r+\left|\xi^{\prime}\right|\right)\right| \geqslant C|R Q|^{1 / 2}\left|\xi^{\prime}\right|^{1 / 2}|r+| \xi^{\prime}||^{1 / 2}$ and the inequalities

$$
\left\{\begin{array}{l}
|Q| \geqslant C\left(a+\left|\alpha^{\prime}\right|^{2}+|\operatorname{Im} q|\right)^{3 / 2}, \\
|R| \geqslant C\left(a+\left|\alpha^{\prime}\right|^{2}+|\operatorname{Im} q|\right)^{1 / 2}, \\
|r+| \alpha^{\prime}||,|R| \leqslant C\left(a+\left|\alpha^{\prime}\right|^{2}+|\operatorname{Im} q|\right)^{1 / 2},
\end{array}\right.
$$


(63) is estimated as follows:

$$
\begin{aligned}
& \left|G_{\mu, \nu}\right| \leqslant \int_{0}^{\infty} \zeta_{3}^{2 \mu+2}|q|^{\mu} e^{-\chi|\operatorname{Im} q| \zeta_{3}^{2}} \mathrm{~d}|\operatorname{Im} q| \\
& \times \int_{\mathbb{R}^{2}} \frac{\left.\zeta_{3}^{\left|v^{\prime}\right|+v_{3}+4}\left|\alpha^{\prime}\right|^{\left|v^{\prime}\right|+1}|a+| \alpha^{\prime}\right|^{2}+\left.|\operatorname{Im} q|\right|^{\nu_{3}+1}}{\left.\zeta_{3}^{2}|a+| \alpha^{\prime}\right|^{2}+\left.|\operatorname{Im} q|\right|^{3 / 2}} e^{-\delta\left|\alpha^{\prime}\right|^{2} \zeta_{3}^{2}} e^{(a-c \sqrt{a}) \zeta_{3}^{2}} \mathrm{~d} \alpha^{\prime} \\
& \leqslant C \zeta_{3}^{2} e^{(a-\sqrt{a}) \zeta_{3}^{2}}
\end{aligned}
$$

Thus, if we choose $a$ sufficiently small, we obtain (62). Combining (61) and (62), we have the desired result. Thus, we have completed the proof of Lemma 4.1.

Now, we estimate the following kernels:

$$
\mathcal{K}_{i, j}(z, t)=\mathcal{L}^{-1}\left(K_{i, j}(z, \lambda)\right), \quad \mathcal{L}_{i, j}(z, t)=\mathcal{L}^{-1}\left(L_{i, j}(z, \lambda)\right), \quad \mathcal{M}_{j}(z, t)=\mathcal{L}^{-1}\left(M_{j}(z, \lambda)\right) .
$$

LEMMA 4.3 The following inequalities hold:

$$
\begin{aligned}
& \left|D_{t}^{\mu} D_{z}^{v} \mathcal{K}_{i, j}(z, t)\right| \leqslant C t^{-\mu-|v| / 2-3 / 2} e^{-c|z|^{2} / t} \\
& \left|D_{t}^{\mu} D_{z}^{v} \mathcal{L}_{i, j}(z, t)\right| \leqslant C t^{-\mu}\left(z_{3}^{2}+t\right)^{-v_{3} / 2}\left(|z|^{2}+t\right)^{-\left(\left|v^{\prime}\right|+2\right) / 2} \\
& \left|D_{t}^{\mu} D_{z}^{v} \mathcal{M}_{j}(z, t)\right| \leqslant C t^{-1 / 2-\mu}\left(|z|^{2}+t\right)^{-(|v|+2) / 2} \quad(i, j=1,2,3) .
\end{aligned}
$$

Proof. Estimate (64) can be obtained in the same manner as the proof of Lemma 4.1.

Let us proceed to the proof of 65 . We estimate only $\mathcal{L}_{1,3}$ because the other kernels can be estimated in the same manner. We can represent $\mathcal{L}_{1,3}$ in the form

where

$$
\mathcal{L}_{1,3}(z, t)=\int_{0}^{z_{3}} \mathrm{~d} y_{3} \int_{\mathbb{R}^{2}} L(z-y, t) D_{y_{3}} E(y) \mathrm{d} y^{\prime},
$$

$$
L(z, t)=\mathcal{F} \mathcal{L}^{-1}\left(\frac{c_{1} i \xi_{1}\left(r^{2}+\left|\xi^{\prime}\right|^{2}\right)}{Q} \frac{c_{1} c_{2}\left|\xi^{\prime}\right|\left(r+\left|\xi^{\prime}\right|\right)}{v\left(R Q+2 c_{1} c_{2}\left|\xi^{\prime}\right|\left(r+\left|\xi^{\prime}\right|\right)\right)} e^{-r z_{3}}\right) \quad \text { and } \quad E(z)=-\frac{1}{4 \pi|z|} .
$$

As in the proof of Lemma 4.1, we can obtain the estimate

Using it, we will obtain

$$
\left|D_{z}^{v} D_{t}^{\mu} L\right| \leqslant C t^{-\mu-|v| / 2-3 / 2} e^{-c|z|^{2} / t} .
$$

$$
\begin{aligned}
\left|D_{z}^{v} D_{t}^{\mu} J\left(z, y_{3}, t\right)\right| & \equiv\left|D_{z}^{v} D_{t}^{\mu} \int_{\mathbb{R}^{2}} L(z-y, t) D_{y_{3}} E(y) \mathrm{d} y^{\prime}\right| \\
& \leqslant C t^{-1 / 2-\mu-v_{3} / 2}\left(|z|^{2}+t\right)^{-\left(\left|v^{\prime}\right|+2\right) / 2} e^{-c\left(z_{3}-y_{3}\right)^{2} / t},
\end{aligned}
$$

by calculations similar to those used in the proof of Lemma 2.2 in [22].

First, we have

$$
\begin{aligned}
D_{z}^{v} D_{t}^{\mu} J\left(z, y_{3}, t\right) \mid & \leqslant C t^{-\mu-|v| / 2-3 / 2} \int_{\mathbb{R}^{2}} e^{-c|z-y|^{2} / t}\left|D_{y_{3}} E(y)\right| \mathrm{d} y^{\prime} \\
& \leqslant C t^{-\mu-|v| / 2-3 / 2} e^{-c\left(Z_{3}-Y_{3}\right)^{2}} .
\end{aligned}
$$

The last inequality is derived by substituting $z / \sqrt{t}=Z, y / \sqrt{t}=Y$. 
On the other hand, through integration by parts, we obtain

$$
\begin{aligned}
D_{z}^{v} D_{t}^{\mu} J\left(z, y_{3}, t\right)= & \int_{B_{\rho / \sqrt{t}}(z)} D_{z_{3}}^{\nu_{3}} D_{t}^{\mu} L(z-y, t) D_{y^{\prime}}^{v^{\prime}} D_{y_{3}} E(y) \mathrm{d} y^{\prime} \\
& +\int_{B_{\rho / \sqrt{t}}(z)^{c}} D_{z}^{v} D_{t}^{\mu} L(z-y, t) D_{y_{3}} E(y) \mathrm{d} y^{\prime} \\
& +\sum_{\left|k^{\prime}\right|+\left|l^{\prime}\right|=\left|\nu^{\prime}\right|-1} \int_{\partial B_{\rho / \sqrt{t}}(z)} D_{z^{\prime}}^{k^{\prime}} D_{z_{3}}^{\nu_{3}} D_{t}^{\mu} L(z-y, t) D_{y^{\prime}}^{l^{\prime}} D_{y_{3}} E(y) \mathrm{d} S \\
\equiv & I_{1}+I_{2}+I_{3},
\end{aligned}
$$

where $\rho=|z|$ and $B_{\rho / \sqrt{t}}(z)=\left\{y^{\prime} \in \mathbb{R}^{2} \mid \sqrt{\left(z^{\prime}-y^{\prime}\right)^{2}+z_{3}^{2}} \leqslant \rho / 2 \sqrt{t}\right\}$. Then we have

$$
\begin{aligned}
& \left|I_{1}\right| \leqslant C t^{-3 / 2-\mu-|v| / 2} \frac{e^{-c\left(Z_{3}-Y_{3}\right)^{2}}}{(\rho / \sqrt{t})^{2+\left|\nu^{\prime}\right|}}, \\
& \left|I_{2}\right| \leqslant C t^{-3 / 2-\mu-|v| / 2} e^{-c|Z-Y|^{2}}, \\
& \left|I_{3}\right| \leqslant C t^{-3 / 2-\mu-|v| / 2}\left(1+\frac{1}{(\rho / \sqrt{t})^{2+\left|\nu^{\prime}\right|}}\right) e^{-c|Z-Y|^{2}} .
\end{aligned}
$$

Combining estimates 680 through 471 , we have

$$
\begin{aligned}
\left|D_{z}^{v} D_{t}^{\mu} J\left(z, y_{3}, t\right)\right| & \leqslant C t^{-3 / 2-\mu-|\nu| / 2}(1+\rho / \sqrt{t})^{-2-\left|\nu^{\prime}\right|} e^{-c\left(Z_{3}-Y_{3}\right)^{2}} \\
& \leqslant C t^{-1 / 2-\mu-v_{3} / 2}\left(|z|^{2}+t\right)^{-1-\left|v^{\prime}\right| / 2} e^{-c\left(z_{3}-y_{3}\right)^{2} / t},
\end{aligned}
$$

which is (67). Hence we can obtain (65) as in the proof of Corollary 1 in [22].

Estimate 667 is obtained in the same manner. Thus, we have proven Lemma 4.3.

Based on the pointwise estimates given in Lemmas 4.1 and 4.3, we can obtain the following estimate:

$$
\sum_{i=1}^{3}\left[\mathcal{L}^{-1}\left(\mathcal{W}_{i}\right)\right]_{q, \mathbb{D}_{\infty}^{3}}^{(2,1)}+\left\|\nabla \mathcal{L}^{-1}(\mathcal{P})\right\|_{q, \mathbb{D}_{\infty}^{3}}+[\theta]_{q, \mathbb{D}_{\infty}^{3}}^{(2,1)} \leqslant C \sum_{i=1}^{3}\left[F_{i}\right]_{q, \mathbb{R}_{\infty}^{2}}^{(1-1 / q, 1 / 2-1 / 2 q)} .
$$

Combining the estimates given in $(56),(55), \sqrt{57})$, and $(72)$, we obtain $(49)$. Thus, the proof of Theorem 4.4 is complete.

Next, we prove Theorem 4.1. We shall find a solution in the form $(\boldsymbol{v}, p, T)=(\boldsymbol{w}+\boldsymbol{u}, \pi+P+q$, $W+U)$, where $(\boldsymbol{w}, \pi), W, P$, and $(\boldsymbol{u}, q, U)$ are solutions to the following problems:

$$
\begin{aligned}
& \left\{\begin{array}{l}
\frac{\partial \boldsymbol{w}}{\partial t}-v \Delta \boldsymbol{w}+c_{0} \nabla \pi=\mathbf{f}, \quad \nabla \cdot \boldsymbol{w}=g \quad \text { in } \Omega_{\infty}, \\
2 v \Pi \mathbf{D}(\boldsymbol{w}) \mathbf{n}=\mathbf{F}, \quad 2 v \mathbf{D}(\boldsymbol{w}) \mathbf{n} \cdot \mathbf{n}-c_{0} \pi=F_{3} \quad \text { on } \Gamma_{\infty}, \\
\boldsymbol{w}=\mathbf{0} \quad \text { on } \Sigma_{\infty},\left.\quad \boldsymbol{w}\right|_{t=0}=\boldsymbol{v}_{0} \quad \text { on } \Omega,
\end{array}\right. \\
& \qquad\left\{\begin{array}{l}
\frac{\partial W}{\partial t}-\chi \Delta W=h \quad \text { in } \Omega_{\infty}, \\
\nabla W \cdot \mathbf{n}=-c_{2} \boldsymbol{w} \cdot \mathbf{n}+G \quad \text { on } \Gamma_{\infty}, \\
W=H \quad \text { on } \Sigma_{\infty},\left.\quad W\right|_{t=0}=T_{0} \quad \text { on } \Omega,
\end{array}\right.
\end{aligned}
$$




$$
\begin{gathered}
\left\{\begin{array}{l}
\Delta P=0 \quad \text { in } \Omega_{\infty}, \\
c_{0} P=-c_{1} W \quad \text { on } \Gamma_{\infty}, \\
\nabla P \cdot \mathbf{n}=0 \quad \text { on } \Sigma_{\infty},
\end{array}\right. \\
\left\{\begin{array}{l}
\frac{\partial \boldsymbol{u}}{\partial t}-v \Delta \boldsymbol{u}+c_{0} \nabla q=-c_{0} \nabla P, \quad \nabla \cdot \boldsymbol{u}=0, \\
\frac{\partial U}{\partial t}-\chi \Delta U=0 \quad \text { in } \Omega_{\infty}, \\
2 v \Pi \mathbf{D}(\boldsymbol{u}) \mathbf{n}=\mathbf{0}, \quad 2 v \mathbf{D}(\boldsymbol{u}) \mathbf{n} \cdot \mathbf{n}-c_{0} p-c_{1} U=0, \\
\nabla U \cdot \mathbf{n}+c_{2} \boldsymbol{u} \cdot \mathbf{n}=0 \quad \text { on } \Gamma_{\infty}, \\
(\boldsymbol{u}, U)=(\mathbf{0}, 0) \quad \text { on } \Sigma_{\infty},\left.\quad(\boldsymbol{u}, U)\right|_{t=0}=(\mathbf{0}, 0) \quad \text { on } \Omega .
\end{array}\right.
\end{gathered}
$$

In the manner described in [19], we can prove that problem $(73)$ has a unique solution $(\boldsymbol{w}, \pi)$ satisfying the following estimate:

$$
\begin{aligned}
\|\boldsymbol{w}\|_{q, \Omega_{\infty}}^{(2,1)}+\|\pi\|_{q, \Omega_{\infty}}^{(1,0)} \leqslant & C\left(\|\mathbf{f}\|_{q, \Omega_{\infty}}+\|g\|_{q, \Omega_{\infty}}^{(1,0)}+\|\mathbf{R}\|_{q, \Omega_{\infty}}^{(0,1)}\right. \\
& \left.+\|\mathbf{F}\|_{q, \Gamma_{\infty}}^{(1-1 / q, 1 / q-1 / 2 q)}+\left\|F_{3}\right\|_{q, \Gamma_{\infty}}^{(1-1 / q, 1 / 2-1 / 2 q)}+\left\|\boldsymbol{v}_{0}\right\|_{q, \Omega}^{(2-2 / q)}\right) .
\end{aligned}
$$

Problems (74) and (75) have unique solutions $W$ and $P$ satisfying

$$
\|W\|_{q, \Omega_{\infty}}^{(2,1)} \leqslant C\left(\|\boldsymbol{w}\|_{q, \Gamma_{\infty}}^{(1-1 / q, 1 / 2-1 / 2 q)}+\|G\|_{q, \Gamma_{\infty}}^{(1-1 / q, 1 / 2-1 / 2 q)}+\|H\|_{q, \Sigma_{\infty}}^{(2-1 / q, 1-1 / 2 q)}+\left\|T_{0}\right\|_{q, \Omega}^{(2-2 / q)}\right)
$$

and

$$
\|P\|_{q, \Omega_{\infty}}^{(2,0)} \leqslant C\|W\|_{q, \Gamma_{\infty}}^{(2-q / 2,1-1 / 2 q)} .
$$

Finally, we consider problem (76). First, using the localization method with the local estimates obtained in Theorems 4.2 through 4.4 , we have

$$
[\boldsymbol{u}]_{q, \Omega_{\infty}}^{(2,1)}+\|\nabla q\|_{q, \Omega_{\infty}}+[U]_{q, \Omega_{\infty}}^{(2,1)} \leqslant C\left(\|\nabla P\|_{q, \Omega_{\infty}}+\|\boldsymbol{u}\|_{q, \Omega_{\infty}}+\|U\|_{q, \Omega_{\infty}}\right) .
$$

Since the derivation is basically analogous to the argument presented in Section 3, we omit it.

The term $\|\boldsymbol{u}\|_{q, \Omega_{\infty}}+\|U\|_{q, \Omega_{\infty}}$ is estimated as follows. From Theorem 3.6, we have

$$
\|\boldsymbol{u}(t)\|_{q, \Omega}+\|U(t)\|_{q, \Omega} \leqslant C \int_{0}^{t} e^{-\gamma(t-\tau)}\|\nabla P(\tau)\|_{q, \Omega} \mathrm{d} \tau
$$

for a positive constant $\gamma$. From this estimate, by using Young's inequality, we obtain

$$
\|\boldsymbol{u}\|_{q, \Omega_{\infty}}+\|U\|_{q, \Omega_{\infty}} \leqslant C\|\nabla P\|_{q, \Omega_{\infty}}
$$

Then we have

$$
\|\boldsymbol{u}\|_{q, \Omega_{\infty}}^{(2,1)}+\|\nabla q\|_{q, \Omega_{\infty}}+\|U\|_{q, \Omega_{\infty}}^{(2,1)} \leqslant C\|\nabla P\|_{q, \Omega_{\infty}} .
$$

Combining the estimates in (77) through 80 , we deduce 43 . Thus, we have completed the proof.

In the following section, we prove the solvability of the nonlinear problem. For this purpose, it is more convenient to restate the above result as the following theorem easily derived from Theorem 4.1 for a sufficiently small $\gamma$. 
THEOREM 4.5 Let $1<q<\infty$. Assume that

$$
\boldsymbol{v}_{0} \in W_{q}^{2-2 / q}(\Omega), \quad T_{0} \in W_{q}^{2-2 / q}(\Omega),
$$

$e^{\gamma t} \mathbf{f} \in L_{q}\left(\Omega_{\infty}\right), \quad e^{\gamma t} g \in W_{q}^{1,0}(\Omega), \quad e^{\gamma t} h \in L_{q}\left(\Omega_{\infty}\right), \quad e^{\gamma t} H \in W_{q}^{2-1 / q, 1-1 / 2 q}\left(\Sigma_{\infty}\right)$,

$e^{\gamma t} \mathbf{F} \in W_{q}^{1-1 / q, 1 / 2-1 / 2 q}\left(\Gamma_{\infty}\right), \quad e^{\gamma t} F_{3} \in W_{q}^{1-1 / q, 1 / 2-1 / 2 q}\left(\Gamma_{\infty}\right), \quad e^{\gamma t} G \in W_{q}^{1-1 / q, 1 / 2-1 / 2 q}\left(\Gamma_{\infty}\right)$, and $g=\nabla \cdot \mathbf{R}, e^{\gamma t} \mathbf{R} \in W_{q}^{1}\left(0, \infty ; L_{q}(\Omega)\right)$. Then problem 42 has a solution

$$
(\boldsymbol{v}, p, T) \in W_{q}^{2,1}\left(\Omega_{\infty}\right) \times W_{q}^{1,0}\left(\Omega_{\infty}\right) \times W_{q}^{2,1}\left(\Omega_{\infty}\right)
$$

satisfying the estimate

$$
\begin{aligned}
& \left\|e^{\gamma t} \boldsymbol{v}\right\|_{q, \Omega_{\infty}}^{(2,1)}+\left\|e^{\gamma t} p\right\|_{q, \Omega_{\infty}}^{(1,0)}+\left\|e^{\gamma t} T\right\|_{q, \Omega_{\infty}}^{(2,1)} \\
& \leqslant \\
& \quad C\left(\left\|e^{\gamma t} \mathbf{f}\right\|_{q, \Omega_{\infty}}+\left\|e^{\gamma t} g\right\|_{q, \Omega_{\infty}}^{(1,0)}+\left\|e^{\gamma t} \mathbf{R}\right\|_{q, \Omega_{\infty}}^{(0,1)}+\left\|e^{\gamma t} h\right\|_{q, \Omega_{\infty}}+\left\|v_{0}\right\|_{q, \Omega}^{(2-2 / q)}+\left\|T_{0}\right\|_{\Omega}^{(2-2 / q)}\right. \\
& \quad+\left\|e^{\gamma t} \mathbf{F}\right\|_{q, \Gamma_{\infty}}^{(1-1 / q, 1 / q-1 / 2 q)}+\left\|e^{\gamma t} F_{3}\right\|_{q, \Gamma_{\infty}}^{(1-1 / q, 1 / 2-1 / 2 q)}+\left\|e^{\gamma t} G\right\|_{q, \Gamma_{\infty}}^{(1-1 / q, 1 / 2-1 / 2 q)} \\
& \left.\quad+\left\|e^{\gamma t} H\right\|_{q, \Sigma_{\infty}}^{(2-1 / q, 1-1 / 2 q)}\right),
\end{aligned}
$$

where $C=C(q, \Omega)$ and $\gamma$ are positive constants.

\section{Nonlinear problem}

In this section, we prove Theorem 2.1. First, let us rewrite problem 8 in the following equivalent form:

$$
\left\{\begin{array}{l}
\frac{\partial \boldsymbol{u}}{\partial t}-v \Delta \boldsymbol{u}+\left(\rho-\rho_{e}\right)^{-1} \nabla q=\mathcal{F}_{1}(\boldsymbol{u}, q), \quad \nabla \cdot \boldsymbol{u}=\mathcal{F}_{2}(\boldsymbol{u}) \\
\frac{\partial U}{\partial t}-\frac{\kappa}{\rho C_{p}} \Delta U=\mathcal{F}_{3}(\boldsymbol{u}, U) \quad \text { in } \Omega_{\infty} \\
2 \nu \Pi \mathbf{D}(\boldsymbol{u}) \mathbf{n}=\mathcal{F}_{4}(\boldsymbol{u}), \\
2 v \mathbf{D}(\boldsymbol{u}) \mathbf{n} \cdot \mathbf{n}-\left(\rho-\rho_{e}\right)^{-1} q-\frac{\rho \rho_{e} l}{\left(\rho-\rho_{e}\right)^{2} T_{c}} U=\mathcal{F}_{5}(\boldsymbol{u}, q, U) \\
\nabla U \cdot \mathbf{n}+\frac{l \rho_{e}}{\kappa} \boldsymbol{u} \cdot \mathbf{n}=\mathcal{F}_{6}(\boldsymbol{u}, U) \quad \text { on } \Gamma_{\infty} \\
\boldsymbol{u}=\mathbf{0}, \quad U=H \quad \text { on } \Sigma_{\infty}, \\
\left.\boldsymbol{u}\right|_{t=0}=\boldsymbol{u}_{0},\left.\quad U\right|_{t=0}=U_{0} \quad \text { on } \Omega
\end{array}\right.
$$

where

$$
\begin{aligned}
\mathcal{F}_{1}(\boldsymbol{u}, q)= & v\left(\nabla_{\boldsymbol{u}}^{2}-\nabla^{2}\right) \boldsymbol{u}-\left(\rho-\rho_{e}\right)^{-1}\left(\nabla_{\boldsymbol{u}}-\nabla\right) q+\rho_{e} \rho^{-1}(\boldsymbol{u} \cdot \nabla) \boldsymbol{u} \\
\mathcal{F}_{2}(\boldsymbol{u})= & -\left(\nabla_{\boldsymbol{u}}-\nabla\right) \cdot \boldsymbol{u} \\
\mathcal{F}_{3}(\boldsymbol{u}, U)= & \frac{\kappa}{\rho C_{p}}\left(\nabla_{\boldsymbol{u}}^{2}-\nabla^{2}\right) U+\rho_{e} \rho^{-1}(\boldsymbol{u} \cdot \nabla) U+\frac{2 v}{C_{p}} \mathbf{D}_{\boldsymbol{u}}(\boldsymbol{u}): \mathbf{D}_{\boldsymbol{u}}(\boldsymbol{u}), \\
\mathcal{F}_{4}(\boldsymbol{u})= & -2 v \Pi\left(\mathbf{D}_{\boldsymbol{u}}(\boldsymbol{u}) \mathbf{n}_{\boldsymbol{u}}-\mathbf{D}(\boldsymbol{u}) \mathbf{n}\right) \\
& +\frac{1}{\rho} \Pi_{\boldsymbol{u}}\left[\boldsymbol{u}\left(\left(1-\frac{\rho_{e}}{\rho}\right) \boldsymbol{u}-\left(\boldsymbol{u} \cdot \mathbf{n}_{\boldsymbol{u}}\right) \mathbf{n}_{\boldsymbol{u}}\right)^{t}\right] \mathbf{n}_{\boldsymbol{u}}
\end{aligned}
$$




$$
\begin{aligned}
\mathcal{F}_{5}(\boldsymbol{u}, q, U)= & -2 v\left(\mathbf{D}_{\boldsymbol{u}}(\boldsymbol{u}) \mathbf{n}_{\boldsymbol{u}} \cdot \mathbf{n}_{\boldsymbol{u}}-\mathbf{D}(\boldsymbol{u}) \mathbf{n} \cdot \mathbf{n}\right) \\
& +\frac{1}{\rho}\left[\boldsymbol{u}\left(\left(1-\frac{\rho_{e}}{\rho}\right) \boldsymbol{u}-\left(\boldsymbol{u} \cdot \mathbf{n}_{\boldsymbol{u}}\right) \mathbf{n}_{\boldsymbol{u}}\right)^{t}\right] \mathbf{n}_{\boldsymbol{u}} \cdot \mathbf{n}_{\boldsymbol{u}} \\
& +\frac{\rho \rho_{e} l}{\left(\rho-\rho_{e}\right)^{2}}\left(\ln \left(U+T_{c}\right)-\ln T_{c}-\frac{U}{T_{c}}\right), \\
\mathcal{F}_{6}(\boldsymbol{u}, U)= & -\left(\nabla_{\boldsymbol{u}} U \cdot \mathbf{n}_{\boldsymbol{u}}-\nabla U \cdot \mathbf{n}\right)+\frac{l \rho_{e}}{\kappa} \boldsymbol{u} \cdot\left(\mathbf{n}_{\boldsymbol{u}}-\mathbf{n}\right) .
\end{aligned}
$$

For $K, \gamma>0$, we set

$$
X_{\gamma, K} \equiv\left\{(\boldsymbol{u}, q, U) \in W_{q}^{2,1}\left(\Omega_{\infty}\right) \times W_{q}^{1,0}\left(\Omega_{\infty}\right) \times W_{q}^{2,1}\left(\Omega_{\infty}\right) \mid\|(\boldsymbol{u}, q, U)\|_{\gamma} \leqslant K\right\},
$$

where $\|(\boldsymbol{u}, q, U)\|_{\gamma} \equiv\left\|e^{\gamma t} \boldsymbol{u}\right\|_{q, \Omega_{\infty}}^{(2,1)}+\left\|e^{\gamma t} q\right\|_{q, \Omega_{\infty}}^{(1,0)}+\left\|e^{\gamma t} U\right\|_{q, \Omega_{\infty}}^{(2,1)}$.

LEMmA 5.1 Let $3<q<\infty$. Then, for arbitrary $(\boldsymbol{u}, q, U) \in X_{\gamma, K}$,

$$
\begin{aligned}
& \left\|e^{\gamma t} \mathcal{F}_{1}(\boldsymbol{u}, q)\right\|_{q, \Omega_{\infty}}+\left\|e^{\gamma t} \mathcal{F}_{2}(\boldsymbol{u})\right\|_{q, \Omega_{\infty}}^{(1,0)}+\left\|e^{\gamma t} \hat{\mathcal{F}}_{2}(\boldsymbol{u})\right\|_{q, \Omega_{\infty}}^{(0,1)}+\left\|e^{\gamma t} \mathcal{F}_{3}(\boldsymbol{u}, U)\right\|_{q, \Omega_{\infty}} \\
& +\left\|e^{\gamma t} \mathcal{F}_{4}(\boldsymbol{u})\right\|_{q, \Gamma_{\infty}}^{(1-1 / q, 1 / 2-1 / 2 q)}+\left\|e^{\gamma t} \mathcal{F}_{5}(\boldsymbol{u}, q, U)\right\|_{q, \Gamma_{\infty}}^{(1-1 / q, 1 / 2-1 / 2 q)}+\left\|e^{\gamma t} \mathcal{F}_{6}(\boldsymbol{u}, U)\right\|_{q, \Gamma \infty}^{(1-1 / q, 1 / 2-1 / 2 q)} \\
& \leqslant C K^{2},
\end{aligned}
$$

where $\hat{\mathcal{F}}_{2}(\boldsymbol{u})=\left(\mathcal{J}^{-1}-I\right) \boldsymbol{u}$ and $C$ is a positive constant that is independent of $\boldsymbol{u}, q, U, \gamma$, and $K$.

Proof. Here, we derive the estimates only for $\mathcal{F}_{1}, \mathcal{F}_{2}$, and $\hat{\mathcal{F}}_{2}$, because the other terms can be estimated in the same manner. In the proof, the notation $\|f\|_{q, r, \Omega_{\infty}}$ denotes the norm defined by $\left(\int_{0}^{\infty}\left(\int_{\Omega}|f(x, t)|^{q} \mathrm{~d} x\right)^{r / q} \mathrm{~d} t\right)^{1 / r}$, and $C$ represents various constants that are independent of $\boldsymbol{u}, q, U, \gamma$, and $K$.

We first estimate the nonlinear terms

$$
\left(\nabla_{\boldsymbol{u}}^{2}-\nabla^{2}\right) \boldsymbol{u}, \quad\left(\nabla_{\boldsymbol{u}}-\nabla\right) q, \quad\left(\nabla_{\boldsymbol{u}}-\nabla\right) \cdot \boldsymbol{u}, \quad\left(\mathcal{J}^{-1}-I\right) \boldsymbol{u},
$$

which are derived from the transformation of coordinates in (7).

For $\left(\nabla_{\boldsymbol{u}}^{2}-\nabla^{2}\right) \boldsymbol{u}$, we have

$$
\begin{aligned}
\| e^{\gamma t}\left(\nabla_{\boldsymbol{u}}^{2}-\right. & \left.\nabla^{2}\right) \boldsymbol{u} \|_{q, \Omega_{\infty}} \\
\leqslant & \left\|e^{\gamma t}\left(\left(\mathcal{J}^{*}-I\right) \nabla \cdot \mathcal{J}^{*} \nabla\right) \boldsymbol{u}\right\|_{q, \Omega_{\infty}}+\left\|e^{\gamma t}\left(\nabla \cdot\left(\mathcal{J}^{*}-I\right) \nabla\right) \boldsymbol{u}\right\|_{q, \Omega_{\infty}} \\
\leqslant & C\left(\left\|\mathcal{J}^{*}-I\right\|_{\infty, \Omega_{\infty}}\left\|D \mathcal{J}^{*}\right\|_{q, \infty, \Omega_{\infty}}\left\|e^{\gamma t} D \boldsymbol{u}\right\|_{\infty, q, \Omega_{\infty}}\right. \\
& +\left\|\mathcal{J}^{*}-I\right\|_{\infty, \Omega_{\infty}}\left\|\mathcal{J}^{*}\right\|_{\infty, \Omega_{\infty}}\left\|e^{\gamma t} D^{2} \boldsymbol{u}\right\|_{q, \Omega_{\infty}} \\
& \left.+\left\|D\left(\mathcal{J}^{*}-I\right)\right\|_{q, \infty, \Omega_{\infty}}\left\|e^{\gamma t} D \boldsymbol{u}\right\|_{\infty, q, \Omega_{\infty}}+\left\|\mathcal{J}^{*}-I\right\|_{\infty, \Omega_{\infty}}\left\|e^{\gamma t} D^{2} \boldsymbol{u}\right\|_{q, \Omega_{\infty}}\right) .
\end{aligned}
$$

Using the estimate

$$
\left\|\int_{0}^{t} D \boldsymbol{u} \mathrm{d} \tau\right\|_{\infty, \Omega_{\infty}} \leqslant C\left(\int_{0}^{\infty} e^{-\gamma q^{\prime} \tau} \mathrm{d} \tau\right)^{1 / q^{\prime}}\left\|e^{\gamma t} \boldsymbol{u}\right\|_{q, \Omega_{\infty}}^{(2,0)} \leqslant C K,
$$


we have $\left\|\mathcal{J}^{*}-I\right\|_{\infty, \Omega_{\infty}},\left\|D\left(\mathcal{J}^{*}-I\right)\right\|_{q, \infty, \Omega_{\infty}},\left\|D \mathcal{J}^{*}\right\|_{q, \infty, \Omega_{\infty}} \leqslant C K$, and $\left\|\mathcal{J}^{*}\right\|_{\infty, \Omega_{\infty}} \leqslant C$, and consequently $\left\|e^{\gamma t}\left(\nabla_{\boldsymbol{u}}^{2}-\nabla^{2}\right) \boldsymbol{u}\right\|_{q, \Omega_{\infty}} \leqslant C K$. In the same manner, $\left\|e^{\gamma t}\left(\nabla_{\boldsymbol{u}}-\nabla\right) q\right\|_{q, \Omega_{\infty}}$, $\left\|e^{\gamma t}\left(\nabla_{\boldsymbol{u}}-\nabla\right) \boldsymbol{u}\right\|_{q, \Omega_{\infty}}^{(1,0)} \leqslant C K$.

The nonlinear term $(\boldsymbol{u} \cdot \nabla) \boldsymbol{u}$ can be estimated as follows:

$$
\|(\boldsymbol{u} \cdot \nabla) \boldsymbol{u}\|_{q, \Omega_{\infty}} \leqslant C\|\boldsymbol{u}\|_{\infty, \Omega_{\infty}}\left\|e^{\gamma t} D \boldsymbol{u}\right\|_{q, \Omega_{\infty}} \leqslant C K^{2}
$$

From these estimates, we obtain the estimates for $\mathcal{F}_{1}$ and $\mathcal{F}_{2}$ as stated above.

Next, let us estimate $\hat{\mathcal{F}}_{2}$. From the estimates $\left\|\frac{\partial}{\partial t}\left(\mathcal{J}^{-1}-I\right)\right\|_{q, \Omega_{\infty}} \leqslant C\|\boldsymbol{u}\|_{q, \Omega_{\infty}}^{(1,0)} \leqslant$ $C\left\|e^{\gamma t} \boldsymbol{u}\right\|_{q, \Omega_{\infty}}^{(1,0)} \leqslant C K$ and $\left\|\mathcal{J}^{-1}-I\right\|_{\infty, \Omega_{\infty}} \leqslant C K$, we have

$$
\begin{aligned}
\left\|\hat{\mathcal{F}}_{2}\right\|_{q, \Omega_{\infty}}^{(0,1)} & \leqslant C\left\|e^{\gamma t}\left(\mathcal{J}^{-1}-I\right) \boldsymbol{u}\right\|_{q, \Omega_{\infty}}^{(0,1)} \\
& \leqslant C\left(\left\|\frac{\partial}{\partial t}\left(\mathcal{J}^{-1}-I\right)\right\|_{q, \Omega_{\infty}}\left\|e^{\gamma t} \boldsymbol{u}\right\|_{\infty, \Omega_{\infty}}+\left\|\mathcal{J}^{-1}-I\right\|_{\infty, \Omega_{\infty}}\left\|\frac{\partial}{\partial t}\left(e^{\gamma t} \boldsymbol{u}\right)\right\|_{q, \Omega_{\infty}}\right) \leqslant C K^{2} .
\end{aligned}
$$

Thus, we have proven the lemma.

In a similar manner, we obtain the following lemma.

LEMmA 5.2 Let $3<q<\infty$. Then, for arbitrary $\left(\boldsymbol{u}_{1}, q_{1}, U_{1}\right),\left(\boldsymbol{u}_{2}, q_{2}, U_{2}\right) \in X_{\gamma, K}$,

$$
\begin{aligned}
\left\|e^{\gamma t}\left(\mathcal{F}_{1}\left(\boldsymbol{u}_{1}, q_{1}\right)-\mathcal{F}_{1}\left(\boldsymbol{u}_{2}, q_{2}\right)\right)\right\|_{q, \Omega_{\infty}}+\left\|e^{\gamma t}\left(\mathcal{F}_{2}\left(\boldsymbol{u}_{1}\right)-\mathcal{F}_{2}\left(\boldsymbol{u}_{2}\right)\right)\right\|_{q, \Omega_{\infty}}^{(1,0)} \\
\quad+\left\|e^{\gamma t}\left(\hat{\mathcal{F}}_{2}\left(\boldsymbol{u}_{1}\right)-\hat{\mathcal{F}}_{2}\left(\boldsymbol{u}_{2}\right)\right)\right\|_{q, \Omega_{\infty}}^{(0,1)}+\left\|e^{\gamma t}\left(\mathcal{F}_{3}\left(\boldsymbol{u}_{1}, U_{1}\right)-\mathcal{F}_{3}\left(\boldsymbol{u}_{2}, U_{2}\right)\right)\right\|_{q, \Omega_{\infty}} \\
\quad+\left\|e^{\gamma t}\left(\mathcal{F}_{4}\left(\boldsymbol{u}_{1}\right)-\mathcal{F}_{4}\left(\boldsymbol{u}_{2}\right)\right)\right\|_{q, \Gamma_{\infty}}^{(1-1 / q, 1 / 2-1 / 2 q)} \\
\quad+\left\|e^{\gamma t}\left(\mathcal{F}_{5}\left(\boldsymbol{u}_{1}, q_{1}, U_{1}\right)-\mathcal{F}_{5}\left(\boldsymbol{u}_{2}, q_{2}, U_{2}\right)\right)\right\|_{q, \Gamma_{\infty}}^{(1-1 / q, 1 / 2-1 / 2 q)} \\
\quad+\left\|e^{\gamma t}\left(\mathcal{F}_{6}\left(\boldsymbol{u}_{1}, U_{1}\right)-\mathcal{F}_{6}\left(\boldsymbol{u}_{2}, U_{2}\right)\right)\right\|_{q, \Gamma_{\infty}}^{(1-1 / 2,1 / 2-1 / 2 q)} \leqslant C K\left\|\left(\boldsymbol{u}_{1}-\boldsymbol{u}_{2}, q_{1}-q_{2}, U_{1}-U_{2}\right)\right\|_{\gamma},
\end{aligned}
$$

where $C$ is a positive constant that is independent of $\boldsymbol{u}_{1}, \boldsymbol{u}_{2}, q_{1}, q_{2}, U_{1}, U_{2}, \gamma$, and $K$.

Now, we define the mapping $F$ that maps $(\boldsymbol{u}, q, U) \in X_{\gamma, K}$ to the solution $(\tilde{\boldsymbol{u}}, \tilde{q}, \tilde{U})$ of the problem $\mathcal{L}(\tilde{\boldsymbol{u}}, \tilde{q}, \tilde{U})=\mathcal{F}(\boldsymbol{u}, q, U)$, where $\mathcal{L}(\boldsymbol{u}, q, U)$ and $\mathcal{F}(\boldsymbol{u}, q, U)$ represent the left-hand side and the right-hand side, respectively, of 82 .

Lemmas 5.1 and 5.2 indicate that $F$ is a contraction on $X_{\gamma, K}$ for suitably chosen $K$.

From Theorem 4.1 and Lemma 5.1, we have

$$
\|(\tilde{\boldsymbol{u}}, \tilde{q}, \tilde{U})\|_{\gamma} \leqslant C\left(\left\|\boldsymbol{u}_{0}\right\|_{q, \Omega}^{(2-2 / q)}+\left\|U_{0}\right\|_{q, \Omega}^{(2-2 / q)}+\|H\|_{q, \Sigma_{\infty}}^{(2-1 / q, 1-1 / 2 q)}+K^{2}\right) .
$$

Thus, if we choose $K \leqslant 1 / 2 C$, then for arbitrary $\left(\boldsymbol{u}_{0}, U_{0}, H\right)$ satisfying $\left\|\boldsymbol{u}_{0}\right\|_{q, \Omega}^{(2-2 / q)}+$ $\left\|U_{0}\right\|_{q, \Omega}^{(2-2 / q)}+\|H\|_{\Sigma_{\infty}}^{(2-1 / q, 1-1 / 2 q)} \leqslant K^{2}$, we have the estimate

$$
\|(\tilde{\boldsymbol{u}}, \tilde{q}, \tilde{U})\|_{\gamma} \leqslant K
$$

This implies that $F$ maps $X_{\gamma, K}$ to itself. 
Next, let us consider the equation

$$
\mathcal{L}\left(\tilde{\boldsymbol{u}}_{1}-\tilde{\boldsymbol{u}}_{2}, \tilde{q}_{1}-\tilde{q}_{2}, \tilde{U}_{1}-\tilde{U}_{2}\right)=\mathcal{F}\left(\boldsymbol{u}_{1}, q_{1}, U_{1}\right)-\mathcal{F}\left(\boldsymbol{u}_{2}, q_{2}, U_{2}\right),
$$

where $\left(\boldsymbol{u}_{1}, q_{1}, U_{1}\right)$ and $\left(\boldsymbol{u}_{2}, q_{2}, U_{2}\right)$ are arbitrary elements of $X_{\gamma, K}$. By the same argument as above, considering Lemma 5.2 and Theorem 4.5, we have the estimate

$$
\left\|\left(\tilde{\boldsymbol{u}}_{1}-\tilde{\boldsymbol{u}}_{2}, \tilde{q}_{1}-\tilde{q}_{2}, \tilde{U}_{1}-\tilde{U}_{2}\right)\right\|_{\gamma} \leqslant C K\left\|\left(\boldsymbol{u}_{1}, q_{1}, U_{1}\right)-\left(\boldsymbol{u}_{2}, q_{2}, U_{2}\right)\right\|_{\gamma} .
$$

Thus, if we choose $K<1 / 2 C$, then $F$ is a contraction on $X_{\gamma, K}$.

Therefore, from the contraction mapping principle, we can obtain the solution of problem $(82)$. Thus, we have completed the proof of Theorem 2.1 .

\section{REFERENCES}

1. Bazalĭ, B. V., \& Degtyarev, S. P. On classical solvability of the multidimensional Stefan problem for convective motion of a viscous incompressible fluid. Mat. Sb. (N.S.) 132 (1987), 3-19 (in Russian); English transl.: Math. USSR-Sb. 60 (1988), 1-17. Zbl 0711.35098 MR 0883909

2. Cannon, J. R., DiBenedetto, E., \& Knightly, G. K. The bidimensional Stefan problem with convection: the time dependent case. Comm. Partial Differential Equations 8 (1983), 1549-1604. Zbl 0547.35117 MR 0728873

3. Desch, W., Hieber, M., \& Prüss, J. $L^{p}$-Theory of the Stokes equation in a half space. J. Evol. Equations 1 (2001), 115-142. Zbl 0983.35102 MR 1838323

4. DiBenedetto, E., \& Friedman, A. Conduction-convection problem with a change of phase. J. Differential Equations 62 (1986), 129-185. Zbl 0593.35085 MR 0833415

5. DiBenedetto, E., \& O'Lealy, M. Three-dimensional conduction-convection problem with change of phase. Arch. Ration. Mech. Anal. 123 (1993), 99-116. Zbl 0802.76084 MR 1219419

6. FARWIG, S., \& SoHR, H. Generalized resolvent estimates for the Stokes system in bounded and unbounded domains. J. Math. Soc. Japan 46 (1994), 607-643. Zbl 0819.35109 MR 1291109

7. Joseph, D. D., \& Renardy, Y. Y. Fundamentals of Two-Fluid Dynamics, Part I. Springer (1992). Zbl 0784.76002 MR 1200237

8. Koss, M. B., Lacombe, J. C., Chait, A., Pines, V., Zlatokowski, M., Glicksman, M. E., \& KAR, P. The Clapeyron effect in succinonitrile: application to crystal growth. J. Cryst. Growth 194 (1998), 143-148.

9. Kusaka, Y., \& TANi, A. Classical solvability of the two-phase Stefan problem in a viscous incompressible fluid flow. Math. Models Methods Appl. Sci. 12 (2002), 365-391. Zbl 1040.35151 MR 1894258

10. Ladyženskaja, O. A., Solonnikov, V. A., \& Ural'Ceva, N. N. Linear and Quasilinear Equations of Parabolic Type. Transl. Math. Monogr. 23, Amer. Math. Soc., Providence (1967). Zbl 0174.15403 MR 0241821

11. Lacombe, J. C., Koss, M. B., Tennenhouse, L. A., Winsa, E. A., \& Glicksman, M. E. Pressure-mediated effects on thermal dendrites. J. Cryst. Growth 279 (2005), 170-185.

12. Maremonti, P., \& Solonnikov, V. A. On nonstationary Stokes problem in exterior domains. Ann. Scuola Norm. Sup. Pisa Cl. Sci. 24 (1997), 395-449. Zbl 0958.35103 MR 1612381

13. MeǏrmanov, A. M. The Stefan Problem. Walter de Gruyter, Berlin (1992). Zbl 0751.35052 MR 1154310

14. Morioka, S., \& IshiI, R. Fundamental equations. In: Fluid Dynamics of Multiphase Flow, S. Morioka (ed.), Asakura Shoten, Tokyo (1991), 59-87 (in Japanese). 
15. Rubinstein, L. I. The Stefan Problem. Transl. Math. Monogr. 27, Amer. Math. Soc., Providence (1971). Zbl 0219.35043 MR 0351348

16. Sawada, T., Takemura, K., Shigematsu, K., Yoda, S., \& Kawasaki, K. Dynamic pressure control for solution growth and its microgravity application. J. Cryst. Growth 158 (1996), 328-335.

17. Shibata, Y., \& Shimizu, S. On a resolvent estimate for the Stokes system with Neumann boundary condition. Differential Integral Equations 16 (2003), 385-426. Z Zbl 1054.35056 MR 1972873

18. Shibata, Y., \& Shimizu, S. On a free boundary problem for the Navier-Stokes equations. Differential Integral Equations 20 (2007), 241-276. MR 2293985

19. SHIMADA, R. On the $L_{p}-L_{q}$ maximal regularity for Stokes equations with Robin boundary condition in a bounded domain. Math. Methods Appl. Sci. 30 (2007), 257-289. Zbl 1107.76029 MR 2285430

20. Solonnikov, V. A. On boundary value problems for linear parabolic systems of differential equations of general form. Trudy Mat. Inst. Steklov. 83 (1965) (in Russian); English transl.: Proc. Steklov Inst. Math. 83 (1965). Zbl 0164.12502 MR 0211083

21. Solonnikov, V. A. Estimates for solutions of nonstationary Navier-Stokes equations. Zap. Nauchn. Sem. LOMI 38 (1973), 153-231 (in Russian); English transl.: J. Soviet Math. 8 (1977), 467-529. Zbl 0404.35081 MR 0415097

22. Solonnikov, V. A. Estimates of solutions of an initial and boundary value problem for the linear nonstationary Navier-Stokes system. Zap. Nauchn. Sem. LOMI 59 (1976), 178-254 (in Russian); English transl.: J. Soviet Math. 10 (1978), 336-393. Z Zbl 0389.76024 MR 0460931

23. Solonnikov, V. A. Solvability of a problem on the motion of a viscous incompressible fluid bounded by a free surface. Izv. Akad. Nauk SSSR Ser. Mat. 41 (1977), 1388-1424 (in Russian); English transl.: Math. USSR-Izv. 11 (1977), 1323-1358. Zbl 0398.76024 MR 0495629

24. Solonnikov, V. A. On the transient motion of an isolated volume of viscous incompressible fluid. Math. USSR-Izv. 31 (1988), 381-405. Zbl 0850.76180 MR 0925094

25. Yamaguti. M., \& Nogi, T. The Stefan Problem. Sangyo Tosho, Tokyo (1978) (in Japanese) 